\title{
Modular curves and Mordell-Weil torsion in F-theory
}

\author{
Nadir Hajouji ${ }^{a}$ and Paul-Konstantin Oehlmann ${ }^{b}$ \\ ${ }^{a}$ Department of Mathematics, University of California, \\ South Hall, Santa Barbara, CA 93106, U.S.A. \\ ${ }^{b}$ Physics Department, Virginia Tech, \\ Robeson Hall 850 West Campus Drive, Blacksburg, VA 24061, U.S.A. \\ E-mail: hajouji@math.ucsb.edu, paul-konstantin.oehlmann@physics.uu.se
}

ABSTRACT: In this work we prove a bound for the torsion in Mordell-Weil groups of smooth elliptically fibered Calabi-Yau 3- and 4-folds. In particular, we show that the set of torsion groups which can occur on a smooth elliptic Calabi-Yau $n$-fold is contained in the set of subgroups which appear on a rational elliptic surface if $n \geq 3$ and is slightly larger for $n=2$. The key idea in our proof is showing that any elliptic fibration with sufficiently large torsion ${ }^{1}$ has singularities in codimension 2 which do not admit a crepant resolution. We prove this by explicitly constructing and studying maps to a modular curve whose existence is predicted by a universal property. We use the geometry of modular curves to explain the minimal singularities that appear on an elliptic fibration with prescribed torsion, and to determine the degree of the fundamental line bundle (hence the Kodaira dimension) of the universal elliptic surface which we show to be consistent with explicit Weierstrass models. The constraints from the modular curves are used to bound the fundamental group of any gauge group $G$ in a supergravity theory obtained from F-theory. We comment on the isolated 8-dimensional theories, obtained from extremal K3's, that are able to circumvent lower dimensional bounds. These theories neither have a heterotic dual, nor can they be compactified to lower dimensional minimal SUGRA theories. We also comment on the maximal, discrete gauged symmetries obtained from certain Calabi-Yau threefold quotients.

KEYwords: Differential and Algebraic Geometry, F-Theory, Global Symmetries

ARXIV EPRINT: 1910.04095

\footnotetext{
${ }^{1}$ By sufficiently large, we mean $\mathbb{Z}_{n}$ with $n \geq 4$ for cyclic torsion, and $\mathbb{Z}_{n} \times \mathbb{Z}_{m}$ with $n+m>4$ in the general case. This condition is necessary to ensure the existence of a fine moduli space.
} 


\section{Contents}

1 Introduction 1

2 Summary of main arguments 3

3 Modular curves $\quad 5$

$\begin{array}{lll}3.1 & \text { General modular curves } & 7\end{array}$

$\begin{array}{llr}3.2 & \text { Cusps } & 9\end{array}$

$\begin{array}{llr}3.3 & \text { Minimal singularities } & 10\end{array}$

$\begin{array}{lll}3.4 & \text { Remarks about the } \mathbb{Z}_{4} \text { case } & 12\end{array}$

4 Bounds on non-simply connected gauge groups from F-theory 13

$\begin{array}{lll}4.1 & \text { F-theory and the role of torsion } & 13\end{array}$

$\begin{array}{lll}4.2 & \text { 6D SUGRA with large putative torsion } & 18\end{array}$

$\begin{array}{lll}4.3 & \text { Higher order and non-prime torsion } & 21\end{array}$

4.4 K3: 8-dimensional exceptions 24

$\begin{array}{ll}4.5 & \text { Bounds on Calabi-Yau quotient torsors } 26\end{array}$

5 Technical results $\quad \mathbf{2 7}$

$\begin{array}{ll}5.1 \text { Global lemmas } & 28\end{array}$

$\begin{array}{lll}5.2 & \text { Local lemmas } & 29\end{array}$

$\begin{array}{lll}5.3 & \text { Summary of technical results } & 30\end{array}$

5.4 Application 31

$\begin{array}{lll}6 & \text { Summary and conclusions } & 32\end{array}$

A Universal elliptic curves with points of order $n \leq 8 \quad 34$

A.1 $n=2,3 \quad 34$

A.2 Universal elliptic curves with a point of order $n \geq 4$

$\begin{array}{ll}\text { A.2.1 Extending } \phi & 36\end{array}$

$\begin{array}{lll}\text { A.3 } & \text { Weierstrass equation for } \mathbb{Z}_{2} \times \mathbb{Z}_{6} & 37\end{array}$

$\begin{array}{lll}\text { A.4 Examples } & 38\end{array}$

$\begin{array}{lll}\text { A.4.1 Explicit equations } & 38\end{array}$

$\begin{array}{ll}\text { B Index of congruence subgroups } & 39\end{array}$

$\begin{array}{lll}\text { B.1 } & \Gamma(n) & 39\end{array}$

$\begin{array}{lll}\text { B.2 } & \Gamma_{1}(n) & 40\end{array}$

B.3 $\Gamma_{0}(n) \quad 41$

B.4 Non-classical congruence groups 41 
C Universal curves for $\mathbb{Z}_{2} \times \mathbb{Z}_{2 m}$ and $\mathbb{Z}_{m} \times \mathbb{Z}_{2 m}$

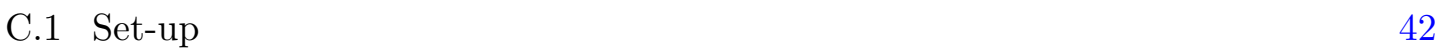

C.2 Construction 43

$\begin{array}{lll}\text { C.3 } & \mathbb{Z}_{2} \times \mathbb{Z}_{4} & 44\end{array}$

\section{Introduction}

Calabi-Yau manifolds play a central role in the description of lower dimensional field and supergravity (SUGRA) theories through their use as compactification backgrounds of String/M/F-theory. Properties of the effective field theories (EFT) such as matter and gauge theory content descend directly from geometric properties of the compactification spaces. The set of all EFTs, that can be obtained via string compactifications is referred to as the string landscape. The string landscape is distinguished from the so-called swampland [1], the set of only seemingly consistent EFTs that do not have a consistent high energy completion together with gravity (for a review see [2]) and therefore not a geometric description as a string compactification. This makes a full classification and study of the string landscape an interesting program both for physics and mathematics. The goal of the program is to find the rules that allow one to distinguish the swampland from the landscape. In the past, these quantum gravity conjectures existed often as folk theorems making use of heuristic black hole arguments, which by themselves might not be fully understood.

In recent years a lot of attention has been given to finding and connecting the set of quantum gravity conjectures and developing tools to put them on more solid grounds, as for example happened for weak gravity [3,4] and distance conjectures [5-7]. These tools make use of the geometric description of physics via Calabi-Yau compactifications and their geometric properties.

F-theory [68] extends the geometrization of physics by also incorporating the type IIB string coupling, enabling descriptions of the largest patch of the string vacua (see e.g. [20]) in a single framework to date. The key idea of F-theory is to focus on elliptically fibered Calabi-Yau manifolds $Y$ and to reformulate the monodromies of $[p, q] 7$-branes that wrap suitable cycles in the base $B$ acting on the IIB axio-dilaton into a geometric action on the complex structure of the elliptic fiber. The gauge algebra living on the branes is encoded in the singularity types of the elliptic fiber and goes beyond the limits of perturbative type IIB.

Certain global aspects of the gauge group $G$ of the F-theory compactifications are encoded in the Mordell-Weil (MW) group $M W(Y / B)$ of the fibration $Y$. For fibrations with a varying axio-dilaton, the MW group admits a decomposition:

$$
M W(Y / B)=\mathbb{Z}^{r} \times T,
$$

where either $T \cong \mathbb{Z}_{n}$ or $T \cong \mathbb{Z}_{n} \times \mathbb{Z}_{m}$ for some integers $n, m, r$. We refer to $T$ is the torsion subgroup of the Mordell-Weil group. A priori, ${ }^{1}$ there are no constraints on the possibilities

\footnotetext{
${ }^{1}$ That is, without making assumptions about the canonical bundle of the total space.
} 
for $n, m, r$ : any combination can, in principle, be obtained by pulling back the fibration along a suitable map $B^{\prime} \rightarrow B$. Classical results on elliptic surfaces show that assumptions about the canonical bundle of the total space constrain the possibilities for the Mordell-Weil group when $\operatorname{dim} Y=2$. In that case, a complete classification of Mordell-Weil groups and configurations of singular fibers is known for rational elliptic surfaces and for elliptically fibered K3s (see e.g. [78-80] as well as [81] and references therein). A table with the torsion subgroups that occur on rational surfaces and K3 surfaces can be found in [32]. There are no analogous classifications for smooth Calabi-Yau elliptic 3-folds and higher. The present work is a step into that direction, by putting bounds on the torsion subgroup $T$.

Within M- and F-theory, the rank $r$ of the MW group gives rise to $r \mathrm{U}(1)$ gauge symmetry factors. This has been extensively studied in the context of F-theory [8-10, 31, 47, 51]. Abelian symmetries are in fact quite subtle. To constrain their maximal possible number $r$ with the help of the strong 6-dimensional SUGRA anomalies is only partially possible [11] whereas the range of possible U(1) singlet charges cannot be constrained at all [15]. In this regard, the geometric description of Abelian symmetries via the free part of the MW group allows for a more systematic and consistent exploration of matter charges [12-14, 16, 17] and maximal Abelian factors [69].

On the other hand, the torsion part of the MW group has been given much less attention in the recent literature. In F-theory compactifications, its effect can be understood as a refinement of the co-weight lattice of representations $[52,54,55,58]$ geometrically induced by the torsion Shioda map: the torsion Shioda map is in correspondence to a certain fractional linear combination of Cartan generators of the gauge algebra $\mathcal{G}$ that lives on the world volume of the IIB 7- branes. Intersections with the torsion Shioda map allows to define a central charge for matter representation $\mathbf{R}$ of $\mathcal{G}$ which constrained to be integral valued which modifies the global gauge group $G$. Although in F-theory only the massless matter spectrum is directly visible, one might expect this constraint to apply to the massive sector as well. Such situations can be viewed as the gauging of $\mathcal{G}$ by a discrete symmetry [71] resulting in a non-simply connected gauge group $G$ with fundamental group $\pi_{1}(G)=T$. A similar effect can happen, although of slightly different origin, when additional $\mathrm{U}(1)$ factors embed non-trivially inside the center of other non-Abelian group factors. In the following however we want to focus purely on the non-Abelian case without additional Abelian gauge factors.

With a view on the swampland program, this begs the obvious question, which nonsimply connected groups can be consistently realized in quantum gravity, specifically in F-theory compactifications. In particular one might ask for bounds on the fundamental group $\pi_{1}(G)$, due to the expectation that the string landscape must be finite. ${ }^{2}$

In [54] Aspinwall and Morrison list various Weierstrass models featuring a wide variety of Mordell-Weil torsion subgroups, going up to $\mathbb{Z}_{6}$ for cyclic groups and $\mathbb{Z}_{3} \times \mathbb{Z}_{3}$ in general. From the perspective of pure 6D SUGRA, it appears possible to set up models whose massless spectrum is consistent with (almost) any putative fundamental group $\pi_{1}(G)=\mathbb{Z}_{n}$

\footnotetext{
${ }^{2}$ The set of elliptically fibered CY threefolds, and therefore landscape of 6D F-theory compactifications in fact has been proven to be "bounded", in the sense that there all such threefolds fit into one of finitely many families, see [41]. This fact alone proves finiteness of possible MW groups in elliptic threefolds.
} 
factor, where $n$ goes way beyond six. However, since the massless fields, are only a small sector of the full theory one should be careful to draw conclusions about symmetries of the full theory, without having some good candidate entity that controls it. In F-theory, there exists such an entity in terms of the torsion sections. ${ }^{3}$ In fact, the mere presence of torsion sections forces the existence of a minimal configuration of singularities whose structure often comes as a surprise, especially for higher order torsion.

In this paper, we want to prove that the list of MW torsion models appearing in Aspinwall and Morrison [54] contains every group that can be realized on a smooth, Calabi-Yau $n$-fold with $n=3,4$. This geometric result allows us to put sharp swampland constraints on the fundamental group of gauge groups in SUGRA theories constructed from $[p, q]-7$ branes in F-theory. As a byproduct, we give a new perspective on elliptic fibrations with torsion sections by connecting them to the modular curve of certain congruence subgroups of $\mathrm{SL}(2, \mathbb{Z}) .{ }^{4}$ The modular curve for each torsion group then allows us conveniently read off all minimal singularities and to proof the boundedness in the cases when the $n$-fold becomes non-crepant resolvable. ${ }^{5}$

In a different context, torsion sections in the Mordell-Weil group can be used as a building block to construct the covering geometry of a specific class of smooth, non-simply connected Calabi-Yau quotient torsors [22]. Their associated F-theory physics admits discrete symmetries coupled to superconformal matter and gravity [18, 19, 21] of the same order as the torsion factor of the covering geometry. Therefore, our bounds also translate to bounds on manifolds that can be obtained using those constructions.

This paper is structured as follows: in section 2 we summarize our main mathematical result and sketch the argument. The full proof is deferred to section 5 and aims at the mathematically inclined reader. In section 3 we give a pedagogical review of congruence subgroups, modular curves and their connection to torsion points of elliptic curves. This allows us in section 3.3 to interpret the presence of singular fibers in torsion models directly from properties of the modular curves. In section 4 we interpret our result in terms of swampland constraint on the order of non-simply connected groups within F-theory. We close with a summary and outlook in section 6 .

\section{Summary of main arguments}

To get started, we fix a finite abelian group $T$, and then we try to construct an elliptic fibration whose Mordell-Weil group contains a subgroup isomorphic to $T$.

\footnotetext{
${ }^{3}$ By analyzing the Ramond-Ramond-charges of BPS solitons in IIA dual theories, the authors of [54] also argued for MW torsion to be the origin of non-simply connected gauge groups which to the best of our knowledge is the only source for such an effect.

${ }^{4}$ In a related F-theory context, fibrations with restricted monodromies have also been considered in [23, 24] and recently [25].

${ }^{5}$ In recent years it has been shown, that 3 -folds with certain terminal singularities can give valid F-theory vacua $[88,89]$ with clear SUGRA description. The singularities in our case however lead to codimension one non-minimal singularities in the fiber which do not admit an understood F-theory limit. Work towards that direction has been made in [75] in terms of non-geometric heterotic dual backgrounds.
} 
Note the Mordell-Weil torsion group of any elliptic fibration injects into the MordellWeil torsion group of any fiber. Since the fibers are isomorphic to $S^{1} \times S^{1}$, this means the Mordell-Weil torsion subgroup of an elliptic fibration is isomorphic to a subgroup of $(\mathbb{Q} / \mathbb{Z}) \times(\mathbb{Q} / \mathbb{Z})$. Furthermore, if the Mordell-Weil group of an elliptic fibration is finitely generated, then the torsion subgroup is a finite abelian group which can be generated by 2 elements.

1. Let $T \subset(\mathbb{Q} / \mathbb{Z}) \times(\mathbb{Q} / \mathbb{Z})$ be a finite subgroup. We say that $T$ is sufficiently large if $T$ contains a point of order at least 4 , or $T \cong \mathbb{Z}_{3} \times \mathbb{Z}_{3}$.

For sufficiently large groups $T,{ }^{6}$ it is known that there exists an elliptic surface $S \rightarrow C$, with $M W(S / C)_{\text {tors }} \cong T$, and satisfying the following universal property: for any elliptic fibration $\pi: Y \rightarrow B$ whose Mordell-Weil group contains a subgroup isomorphic to $T$, there are rational maps making the following square commute:

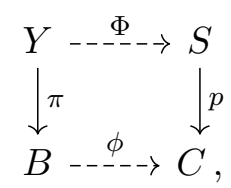

The curve $C$ in the diagram is the modular curve associated to $T$.

A Weierstrass equation for $Y$ can be obtained by pulling back a Weierstrass equation for $S \rightarrow C$ using $\phi$. This reduces the problem of classifying fibrations $Y \rightarrow B$ over a fixed base $B$, with a prescribed torsion group $T$, to understanding rational maps $B \rightarrow C$.

2. Next, we use the Calabi-Yau condition to limit the bases we have to consider. The fundamental line bundle of an elliptic fibration $\pi: Y \rightarrow B$ is the line bundle $\mathcal{L}_{Y / B}=\left(R^{1} \pi_{*} \mathcal{O}_{Y}\right)^{-1}$. We write $\omega_{Y}\left(\right.$ resp. $\left.\omega_{B}\right)$ to denote the canonical bundle of $Y$ (respectively $B$.)

It is known that any Calabi-Yau fibration $Y \rightarrow B$, with $\operatorname{dim} Y \leq 4$, is birational to a fibration $Y_{0} \rightarrow B_{0}$ over a Fano base, i.e. a base where $\omega_{B}^{-1}$ is ample.

This allows us to deduce two things:

- First, if $B$ is a Fano variety and $C$ is a positive genus curve, then every rational map $B \rightarrow C$ is constant. This means we only need to consider torsion groups $T$ for which the associated modular curve has genus 0. By work of Cox and Parry, it is well-known that there are only 19 such groups, so the problem is reduced to determining which of those 19 can occur on a smooth Calabi-Yau.

- Second, if $Y$ is Calabi-Yau and $B$ is Fano, then $\mathcal{L}_{Y / B} \cong \omega_{B}^{-1}$. We show that $\mathcal{L}_{Y / B} \cong \phi^{*}\left(\mathcal{L}_{S / B}\right)$, which further constrains the possibilities for $\phi$. In particular, this allows us to show that $\phi$ can't be a morphism under these assumptions.

\footnotetext{
${ }^{6}$ The only finite subgroups of $(\mathbb{Q} / \mathbb{Z}) \times(\mathbb{Q} / \mathbb{Z})$ which fail to be sufficiently large are $0, \mathbb{Z}_{2}, \mathbb{Z}_{3}, \mathbb{Z}_{2} \times \mathbb{Z}_{2}$. These torsion groups appear in Calabi-Yau $n$-folds of any dimension, so we do not need to consider them to prove our theorem.
} 
3. Finally, we show that for any $b \in B$ where $\phi$ is not defined, the Weierstrass coefficients $(f, g, \Delta)$, defined in (3.1) over $b$ are guaranteed to vanish to order at least $(4 d, 6 d, 12 d)$, where $d$ is a positive integer multiple of $\operatorname{deg} \mathcal{L}_{S / C}$.

It is well known that a crepant resolution of the total space does not exist if the order of vanishing of $(f, g, \Delta)$ is at least $(8,12,24)$ over a codimension 2 locus in $B$. This allows us to conclude that the singular model can be crepantly resolved only if $d=1$. In appendix B, we show how to compute the integer $d$, and find that $d=1$ is only possible if the torsion group is one of the 10 groups shown above.

In appendix A, we show explicitly how to construct the surface $S \rightarrow C$ and the map $\phi$ for $T$ cyclic. In appendix $\mathrm{C}$ we give a construction and proof of the universal property for $S \rightarrow C$ and $\phi$ for groups of the form $\mathbb{Z}_{2} \times \mathbb{Z}_{2 m}$ and $\mathbb{Z}_{m} \times \mathbb{Z}_{2 m}$. In section 4.1 we will then study the application of our main theorem within the physics of F-theory.

\section{Modular curves}

Modular curves play a crucial role in our arguments and are not standard in the physics literature, so we start by giving a brief review of the theory. ${ }^{7}$ In this section, we will describe the geometry of modular curves as analytic objects. In the appendix, we give an algebraic construction for certain families of modular curves, together with equations for associated universal elliptic curves. The analytic perspective described in this section is helpful in explaining the minimal configurations of singularities observed on elliptic fibrations with prescribed torsion. This perspective also allows us to compute the degree of $\mathcal{L}_{S / C}$, even in the absence of a Weierstrass model for the universal surface.

"The" modular curve. The modular curve $X(1)^{\circ}$ parametrizes isomorphism classes of elliptic curves $\mathbb{C}$.

- Algebraically, every elliptic curve admits a short Weierstrass equation:

$$
y^{2}=x^{3}+f x+g, \quad\left(f, g \in \mathbb{C}, \Delta=4 f^{3}+27 g^{2} \neq 0\right) .
$$

Two such elliptic curves, with coefficients $f_{i}, g_{i}, i=1,2$, are isomorphic if and only if there exists $\lambda \in \mathbb{C}^{\times}$such that $f_{1}=\lambda^{4} f_{2}$ and $g_{1}=\lambda^{4} g_{2}$.

Thus, $X(1)^{\circ}$ can be identified with the quotient:

$$
X(1)^{o}=\left\{(f, g) \in \mathbb{C}^{2}: \Delta \neq 0\right\} /\left((f, g) \sim\left(\lambda^{4} f, \lambda^{6} g\right)\right) .
$$

Note that this quotient has two singular points, namely the orbits $[(1,0)],[(0,1)]$, corresponding to the two elliptic curves with complex multiplication.

- Analytically, Riemann's uniformization theorem shows that every elliptic curve is isomorphic, as a Riemann surface, to $\mathbb{C} / \Lambda$, where $\Lambda$ is the lattice of periods of the elliptic curve. Two quotients give rise to isomorphic elliptic curves if and only if the corresponding lattices are homothetic, i.e. scalar multiples of one another.

\footnotetext{
${ }^{7}$ For a more detailed exposition, see [73].
} 


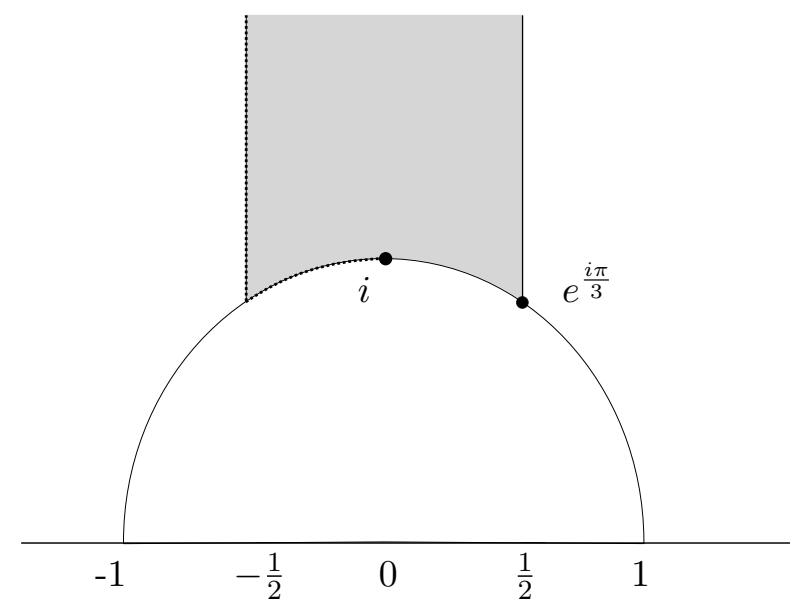

Figure 1. The Fundamental domain of $\tau$ for $X(1)=\mathcal{H} / \operatorname{SL}(2, \mathbb{Z})$.

To compute the moduli space, we assume the lattice has been scaled so that one of the basis vectors is at 1 and the other basis vector lies in the (open) upper half plane, depicted in figure 1 . This allows us to identify points $\tau \in \mathcal{H}$ with isomorphism classes of elliptic curves: the point $\tau$ represents the elliptic curve $\mathbb{C} /(\mathbb{Z} \oplus \mathbb{Z} \tau)$. Two points $\tau, \tau^{\prime} \in \mathcal{H}$ represent the same elliptic curve if and only if they are in the same $\mathrm{SL}(2, \mathbb{Z})$-orbit (here $\mathrm{SL}(2, \mathbb{Z})$ is acting on $\mathcal{H}$ by fractional linear transformations). The region:

$$
\mathcal{D}=\left\{\tau \in \mathcal{H}: 1<|\tau|,-\frac{1}{2}<\operatorname{Re}(\tau) \leq \frac{1}{2}\right\} \cup\left\{e^{i \theta}: \frac{\pi}{3} \leq \theta \leq \frac{\pi}{2}\right\},
$$

is a fundamental domain for the quotient, so points in $\mathcal{D}$ are in bijection with points in $X(1)^{o}$. The orbits of $i$ and $e^{i \pi / 3}$ in $\mathcal{H} / \mathrm{SL}(2, \mathbb{Z})$, correspond to the aforementioned singular orbits $(1,0)$ and $(0,1)$ of $(f, g)$. They represent the square and hexagonal lattices, which have extra symmetries.

The celebrated $j$-function, which is defined using Eisenstein series in the analytic setting or simply as:

$$
j([(f, g)])=1728 \cdot \frac{4 f^{3}}{4 f^{3}+27 g^{2}},
$$

in the algebraic one, gives a bijection between $X(1)^{o}$ and $\mathbb{A}^{1}$.

We can compactify $X(1)^{\circ}$ by adding a single point to the moduli space representing the isomorphism class of an $I_{1}$ curve. Analytically, we achieve this by taking the quotient of the extended upper half plane $\mathcal{H}^{*}=\mathcal{H} \cup \mathbb{Q} \cup\{\infty\}$. The compactified modular curve is denoted $X(1)$, and is isomorphic to $\mathbb{P}^{1}$ as a Riemann surface.

The modular curve $X(1)$ is crucial in the study of general modular curves, as every other modular curve comes equipped with a canonical map to $X(1)$. 


\subsection{General modular curves}

We now consider general modular curves. We start discussing modular curves associated to cyclic torsion groups of order $n$. Formally, these are fine moduli spaces for pairs $(E, p)$ where $E$ is an elliptic curve over $\mathbb{C}$ and $p$ is a point of order exactly $n$ on $E$, where $(E, p),\left(E^{\prime}, p^{\prime}\right)$ are identified whenever there exists an isomorphism $E \rightarrow E^{\prime}$ taking $p$ to $p^{\prime}$. We will construct these modular curves analytically ${ }^{8}$ as quotients of $\mathcal{H}$ by an appropriate subgroup of $\Gamma_{1}(n) \leq S L_{2}(\mathbb{Z})$.

- Every elliptic curve over $\mathbb{C}$ is isomorphic to $E_{\tau}=\mathbb{C} / \Lambda_{\tau}$, where $\Lambda_{\tau}=\tau \mathbb{Z} \oplus \mathbb{Z} \subset \mathbb{C}$ is a rank 2 lattice, and $\tau \in \mathcal{H}$.

- Every point of order $n$ on $E_{\tau}$ has the form $\frac{c \tau+d}{n}+\Lambda_{\tau}$ for some pair of integers $c, d$ satisfying $\operatorname{gcd}(c, d, n)=1$.

Thus, it suffices to study pairs $\left(E_{\tau}, \frac{c \tau+d}{n}\right)$ for $\tau \in \mathcal{H}$ and $c, d \in \mathbb{Z}$ satisfying $\operatorname{gcd}(c, d, n)=1$. We now need to determine when two pairs $\left(E_{\tau}, \frac{c \tau+d}{n}\right),\left(E_{\tau^{\prime}}, \frac{c^{\prime} \tau^{\prime}+d^{\prime}}{n}\right)$ are isomorphic. We will show that the action of $\operatorname{SL}(2, \mathbb{Z})$ on the space of bases of $\Lambda_{\tau}$ induces an action on the $n$-torsion points of $E_{\tau}$.

Let $p \in E_{\tau}$ a point on the elliptic curve, that we can write uniquely as

$$
p: \quad x \tau+y+\Lambda_{\tau} \text { for } x, y \in[0,1) .
$$

For $\gamma=\left(\begin{array}{ll}a & b \\ c & d\end{array}\right) \in \operatorname{SL}(2, \mathbb{Z})$, we define the action on that point $p$ as

$$
\gamma \cdot p=(a \tau+b) x+(c \tau+d) y+\Lambda_{\tau} .
$$

It's clear that $\gamma$ induces an automorphism of $E_{\tau}$ as a group, so it necessarily restricts to an automorphism of the $n$-torsion of $E_{\tau}$. Thus $\mathrm{SL}(2, \mathbb{Z})$ acts on $n$-torsion pairs $\left(E_{\tau}, \frac{c \tau+d}{n}\right)$. We next show that it acts transitively on such pairs: in other words, for any pair $\left(E_{\tau}, p+\Lambda_{\tau}\right)$, we can find $\gamma \in \operatorname{SL}(2, \mathbb{Z})$ such that $\gamma^{-1} \cdot p+\Lambda_{\tau}=\frac{1}{n}+\Lambda_{\tau}$. This will show that the moduli space of pairs $\left(E, p+\Lambda_{\tau}\right)$ is isomorphic to $\mathcal{H} / \Gamma_{1}(n)$, where $\Gamma_{1}(n)$ is the stabilizer of the pair $\left(E_{\tau}, \frac{1}{n}+\Lambda_{\tau}\right)$. Let $\frac{c \tau+d}{n}+\Lambda_{\tau}$ be an arbitrary point of order $n$. Since $\operatorname{gcd}(c, d, n)=1$, there exist integers $a, b, k$ such that $a d-b c+k n=1$. That is equivalent to saying there is a matrix in $\operatorname{SL}\left(2, \mathbb{Z}_{n}\right)$ with entries congruent to $\gamma^{\prime}$ with entries as $a d-b c+k n=1$. The reduction map $\mathrm{SL}(2, \mathbb{Z}) \rightarrow \mathrm{SL}\left(2, \mathbb{Z}_{n}\right)$ is surjective, so there exists $\gamma \in \mathrm{SL}(2, \mathbb{Z})$ such that $\gamma \cong\left(\begin{array}{ll}a & b \\ c & d\end{array}\right) \quad(\bmod n)$. Observe now the inverse action of $\gamma$ on the pair

$$
\gamma^{-1} \cdot\left(E_{\tau}, \frac{c \tau+d}{n}+\Lambda_{\tau}\right)=\left(E_{\tau}, \frac{c(d \tau-b)+d(-c \tau+a)}{n}+\Lambda_{\tau}\right)=\left(E_{\tau}, \frac{1}{n}+\Lambda_{\tau}\right) .
$$

Thus, if we take a larger fundamental domain in $\mathcal{H}$, we do not have to keep track of the specific coefficients $c, d$ for the point of order $n$. The problem is now reduced to understanding the stabilizer of $\left(E_{\tau}, \frac{1}{n}+\Lambda_{\tau}\right)$.

\footnotetext{
${ }^{8}$ The universal property ensures that the fine moduli space is unique up to isomorphism, if it exists, which is why the analytic construction gives the same modular curve as the algebraic construction.
} 
a.)

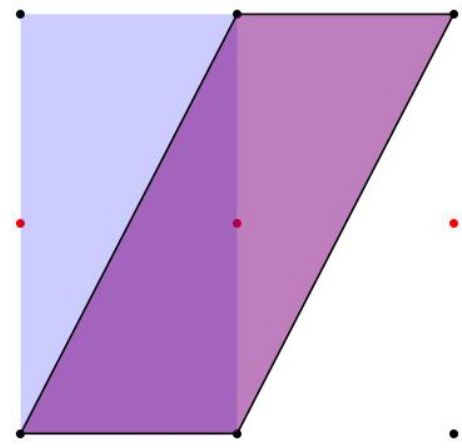

b.)

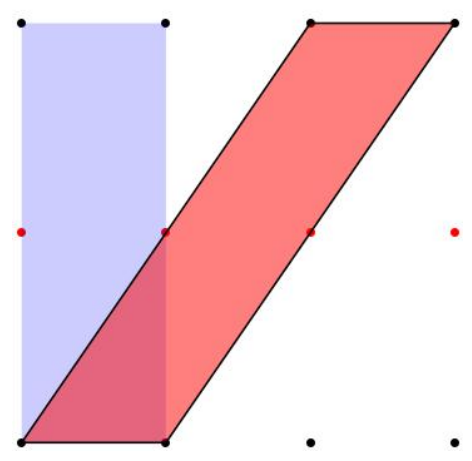

Figure 2. Above we have chosen a fundamental domain colored in blue for $E_{\tau}$ the depiction of a 2 -torsion point $\frac{\tau}{2}+\Lambda_{\tau}$ in orange. We act on the basis by the generator $T$ in $a$.) which translates the point $\frac{\tau}{2}+\Lambda$ to $\frac{\tau+1}{2}+\Lambda$. In $b$.) we act on the fundamental domain by $T^{2}$ in to obtain the pink one while fixing the torsion point.

Specifically, we want a characterization of those $\gamma \in \operatorname{SL}(2, \mathbb{Z})$ that fix the torsion point

$$
\gamma \cdot \frac{1}{n}+\Lambda_{\tau}=\frac{1}{n}+\Lambda_{\tau}
$$

The new basis is given by $a \tau+b, c \tau+d$, and $\frac{1}{n}+\Lambda_{\tau}$ is being mapped to $\frac{c}{n} \tau+\frac{d}{n}+\Lambda_{\tau}$. It is clear that $\frac{1}{n}$ is fixed exactly when $\frac{c}{n} \in \mathbb{Z}$ and $\frac{d}{n} \in \frac{1}{n}+\mathbb{Z}$. An illustration of this action on the torus lattice is depicted in figure 2 for a chosen order two torsion point. In particular, we need the entries to satisfy

$$
c \equiv 0 \quad(\bmod n) \quad \text { and } \quad d \equiv 1 \quad(\bmod n)
$$

It then follows, that the matrices need to satisfy

$$
\left(\begin{array}{ll}
a & b \\
c & d
\end{array}\right) \equiv\left(\begin{array}{ll}
* & * \\
0 & 1
\end{array}\right) \quad(\bmod n) \equiv\left(\begin{array}{ll}
1 & * \\
0 & 1
\end{array}\right) \quad(\bmod n)
$$

where we have used $a d-b c=1$ and $c \equiv 0(\bmod n)$ such that we must have $a d \equiv a \equiv 1$ $(\bmod n)$.

To summarize, a matrix $\left(\begin{array}{ll}a & b \\ c & d\end{array}\right) \in \mathrm{SL}(2, \mathbb{Z})$ fixes $\left(E_{\tau}, \frac{1}{n}+\Lambda_{\tau}\right)$ as a torsion pair if and only if

$$
\left(\begin{array}{ll}
a & b \\
c & d
\end{array}\right) \equiv\left(\begin{array}{ll}
1 & * \\
0 & 1
\end{array}\right) \quad(\bmod n)
$$

We thus define:

$$
\Gamma_{1}(n)=\left\{\gamma \in \operatorname{SL}(2, \mathbb{Z}): \gamma \equiv\left(\begin{array}{ll}
1 & * \\
0 & 1
\end{array}\right) \quad(\bmod n)\right\}
$$


We can then go ahead and define the (open) modular curve as the quotient $X_{1}(n)^{o}=$ $\mathcal{H} / \Gamma_{1}(n) .{ }^{9}$ We identify points on $X_{1}(n)^{o}$ with pairs consisting of an elliptic curve $E_{\tau}$ and a point of order $n$. The compactified modular curve $X_{1}(n)$ is obtained by taking the quotient of the extended upper half plane by $\Gamma_{1}(n)$. The cusps of the modular curve are the points in $X_{1}(n) \backslash X_{1}(n)^{o}$.

There are also other examples of modular curves that appear frequently in the literature. These are denoted $X(n)$ and $X_{0}(n)$, and are defined as quotients of the extended upper half-plane by the subgroups:

$$
\begin{gathered}
\Gamma(n)=\left\{\gamma \in \operatorname{SL}(2, \mathbb{Z}): \gamma \equiv\left(\begin{array}{ll}
1 & 0 \\
0 & 1
\end{array}\right) \quad(\bmod n)\right\}, \\
\Gamma_{0}(n)=\left\{\gamma \in \operatorname{SL}(2, \mathbb{Z}): \gamma \equiv\left(\begin{array}{l}
* * \\
0 *
\end{array}\right) \quad(\bmod n)\right\} .
\end{gathered}
$$

As moduli spaces, $X(n)$ classifies pairs $\left(E,\left(p_{1}, p_{2}\right)\right)$ where $p_{1}, p_{2}$ are "suitable" 10 generators of the $n$-torsion subgroup of $E$, and $X_{0}(n)$ classifies pairs $(E,\langle p\rangle)$ where $\langle p\rangle$ is a cyclic subgroup of order $n$.

Finally, we remark that while a lot has been written on the modular curves $X_{1}(n), X(n), X_{0}(n)$, much less has been written about the modular curves associated to "generic" torsion groups. We relied on ad hoc methods to analyze and rule out fibrations with torsion groups which are not cyclic or isomorphic to $\mathbb{Z}_{m} \times \mathbb{Z}_{m}$. See appendix C for details.

\subsection{Cusps}

Finally, we need to define the width of a cusp, as this idea plays an important role to discuss minimal singularities in section 3.3. To that end, we introduce the following notation. Set:

$$
\operatorname{SL}(2, \mathbb{Z})_{\infty}=\left\{\left(\begin{array}{ll}
1 & n \\
0 & 1
\end{array}\right): n \in \mathbb{Z}\right\} .
$$

This is the stabilizer subgroup of $\infty$ for the action on $\mathcal{H}^{*}$ by fractional linear transformations.

For a general subgroup $\Gamma \subset \mathrm{SL}(2, \mathbb{Z})$, we define:

$$
\Gamma_{\infty}=\Gamma \cap \operatorname{SL}(2, \mathbb{Z})_{\infty}=\left\{\gamma \in \Gamma: \gamma=\left(\begin{array}{ll}
1 & a \\
0 & 1
\end{array}\right): n \in \mathbb{Z}\right\},
$$

\footnotetext{
${ }^{9}$ This notation is not standard. In the modular curves literature, the curve we are denoting as $X_{1}(n)^{o}$ is usually denoted $Y_{1}(n)$. We've deviated from the standard notation to avoid confusion with the Calabi-Yau total spaces, which we are denoting $Y$.

${ }^{10}$ Technically, $X(n)$ only classifies pairs where $p_{1}, p_{2}$ have a prescribed value under the Weil pairing. This is unavoidable, as any isomorphism of elliptic curves $E \rightarrow E^{\prime}$ takes pairs of $n$-torsion points on $E$ to pairs of $n$-torsion points on $E^{\prime}$ with the same value under the Weil pairing. However, this is sufficient for our purposes, as we can change of value of the Weil pairing by replacing $\left(E,\left(p_{1}, p_{2}\right)\right)$ by $\left(E,\left(p_{1}, k p_{2}\right)\right)$ for a suitable integer $k$ prime to $n$.
} 
Now, fix a congruence subgroup $\Gamma$. Each cusp of the associated modular curve corresponds to the orbit of some $s \in \mathbb{Q}$ under $\Gamma$. Choose such an $s$, and choose $\delta \in \operatorname{SL}(2, \mathbb{Z})$ such that $\delta \cdot s=\infty$. The width of the cusp $x$, denoted $h(x)$, is abstractly defined as:

$$
h(x)=\left[\mathrm{SL}(2, \mathbb{Z})_{\infty}:\left(\delta\{ \pm I\} \Gamma \delta^{-1}\right)_{\infty}\right]
$$

This number encodes the smallest integer $h$ such that $\left(\begin{array}{ll}1 & h \\ 0 & 1\end{array}\right) \in \delta \Gamma \delta^{-1}$; geometrically, we have acted on the upper half plane to put $s$ at the "top" of the picture, and we're counting the number of vertical strips in the fundamental domain. We can also compute the width using the triangulation we pull back from $X(1)$, by dividing the number of triangles that meet at a given cusp by 2 . We will discuss several concrete examples in the following sections.

From the moduli perspective, the width of the cusp encodes the ramification over the $I_{1}$ point on $X(1)$, and thus determines the minimal singularities on the Néron model of the universal elliptic curve. Specifically, whenever there is a cusp of width $h$ on the modular curve, the associated elliptic surface should have an $I_{h}$ singularity (or worse). ${ }^{11}$ This will be discussed further in the minimal singularities section.

\subsection{Minimal singularities}

When considering explicit Weierstrass models we find (e.g. see section 4) that the presence of torsion in $M W$ imposes a minimal number of singular fibers. In the context of F-theory this is surprising, as it forces a minimal gauge group when the full fibration admits torsion. This is particularly true for larger order torsion, where not one but multiple singular fibers/gauge algebra factors are introduced. For example, any fibration with a section of order 5 necessarily has at least two fibers of type $I_{5}$ (or possibly $I_{5 d}$ for some integer $d$ ). For fibrations with 7 -torsion, the number of $I_{7}$ fibers has to be at least 3 . The exact configuration of fibers which is imposed can be computed from the Weierstrass equation of the universal elliptic curve (e.g. see appendix (A)), but obtaining such equations can often be tedious.

Fortunately, the degenerate fibers and the Euler characteristic of the universal elliptic surface $S \rightarrow C$ can both be read off directly from the associated congruence subgroup. Geometrically, the singularities appear because of the cusps on the compactified modular curve. The information needed to determine which singular fibers appear in $S \rightarrow C$ is encoded in the cusps of the modular curve:

- The points on the discriminant locus are in bijection with cusps on the compactified modular curve.

- The fiber over a point in the discriminant locus is of type $I_{d}$, where $d$ is the width of the associated cusp.

Both the number of cusps and their widths can easily be computed using the standard triangulations of the modular curves: the cusps are the points on the real line, and the

\footnotetext{
${ }^{11}$ These points must be of $I_{h}$ type, since $f$ and $g$ are non-vanishing, as they come from special points in the fundamental domain of $X(1)$, that are not mapped onto the real axis.
} 
width of each cusp can be computed by dividing the number of triangles meeting at the cusp by 2. They can also be computed using algebraic methods, see e.g. [73].

The number of triangles in the triangulation is equal to twice the sum of the widths. As a result, it can be interpreted as twice the degree of the discriminant of an elliptic fibration with only the minimal singularities forced by the modular curve. The degree of the discriminant is useful, because it determines the degree of the fundamental line bundle of the universal surface, which is crucial to our analysis.

Since the triangulation is pulled back from the map $X \rightarrow X(1)$, the number of triangles is exactly the degree of the map, which is the index $[\operatorname{SL}(2, \mathbb{Z}): \Gamma]$.

In appendix $\mathrm{B}$, we derive formulas for the index of all congruence subgroups. For example, the index of $\Gamma_{1}(n)$ is:

$$
\left[\Gamma(1): \Gamma_{1}(n)\right]=n^{2} \prod_{p \mid n} 1-\frac{1}{p^{2}},
$$

where the product is taken over all primes dividing $n$. One can check (see appendix B) that this index is equal to 12 when $n=4$ and is divisible by 24 for all $n>4$. For $n>4$, the index can be used to compute the degree of $\mathcal{L}_{S / C}$, where $S \rightarrow C$ is the Néron model of the universal elliptic curve:

$$
\left[\Gamma(1): \Gamma_{1}(n)\right]=24 \operatorname{deg} \mathcal{L}_{S / C}
$$

Thus, when $C=X_{1}(n)$, we have $\operatorname{deg} \mathcal{L}_{S / C}=1$ for $n=5,6$, $\operatorname{deg} \mathcal{L}_{S / C}=2$ for $n=7,8$, $\operatorname{deg} \mathcal{L}_{S / C}=3$ for $n=9,10$, etc. This matches up exactly with the tables in [32], and confirms that the $\mathbb{Z}_{5}, \mathbb{Z}_{6}$ rational elliptic surfaces are extremal. ${ }^{12}$ Similarly, for torsion groups $\mathbb{Z}_{7}, \mathbb{Z}_{8}$, one can check that the $\mathrm{K} 3$ surfaces are extremal. The surfaces associated to torsion groups of larger order have Kodaira dimension 1 and hence are not Calabi-Yau. E.g. the triangulation for $X_{1}(6)$ and $X_{1}(7)$ are depicted in figure 3 using Sage [82, 83] and $X_{1}(6)$ admits the following cusp points

$$
S_{\text {cusp }}^{(6)}:\left\{0^{(6)}, 1 / 3^{(2)}, 1 / 2^{(3)}, \infty^{(1)}\right\},
$$

with their respective widths denoted as superscripts. This means the universal surface should have fibers of type $I_{6}, I_{2}, I_{3}, I_{1}$. These are exactly the discriminant loci, that we also find in the generic Weierstrass model that we discuss in section 4.3.

Similarly, for $X_{1}(7)$, we find that there are 6 cusps (including $\infty$ ) at

$$
S_{\text {cusp }}^{(7)}:\left\{0^{(7)}, 2 / 7^{(1)}, 1 / 3^{(7)}, 3 / 7^{(1)}, 1 / 2^{(7)}, \infty^{(1)}\right\},
$$

three of which have width 7 and three of width 1 , so the universal surface has $3 I_{7}$ fibers and $3 I_{1}$ fibers. This explains why we cannot have a model with only 7 -torsion section but only a single $I_{7}$ fiber. On the contrary the cusp counting of $X_{1}(7)$ correctly predicts all three $I_{7}$ fibers in the K3 (see section 4.4).

\footnotetext{
${ }^{12}$ An elliptic surface $S$ is extremal if $\operatorname{Pic}(S)=h^{1,1}$ and its MW group is finite. This is equivalent to $\operatorname{Pic}(S)=10$ for rational elliptic surfaces $\operatorname{Pic}(S)=20$ for elliptic K3 surfaces.
} 

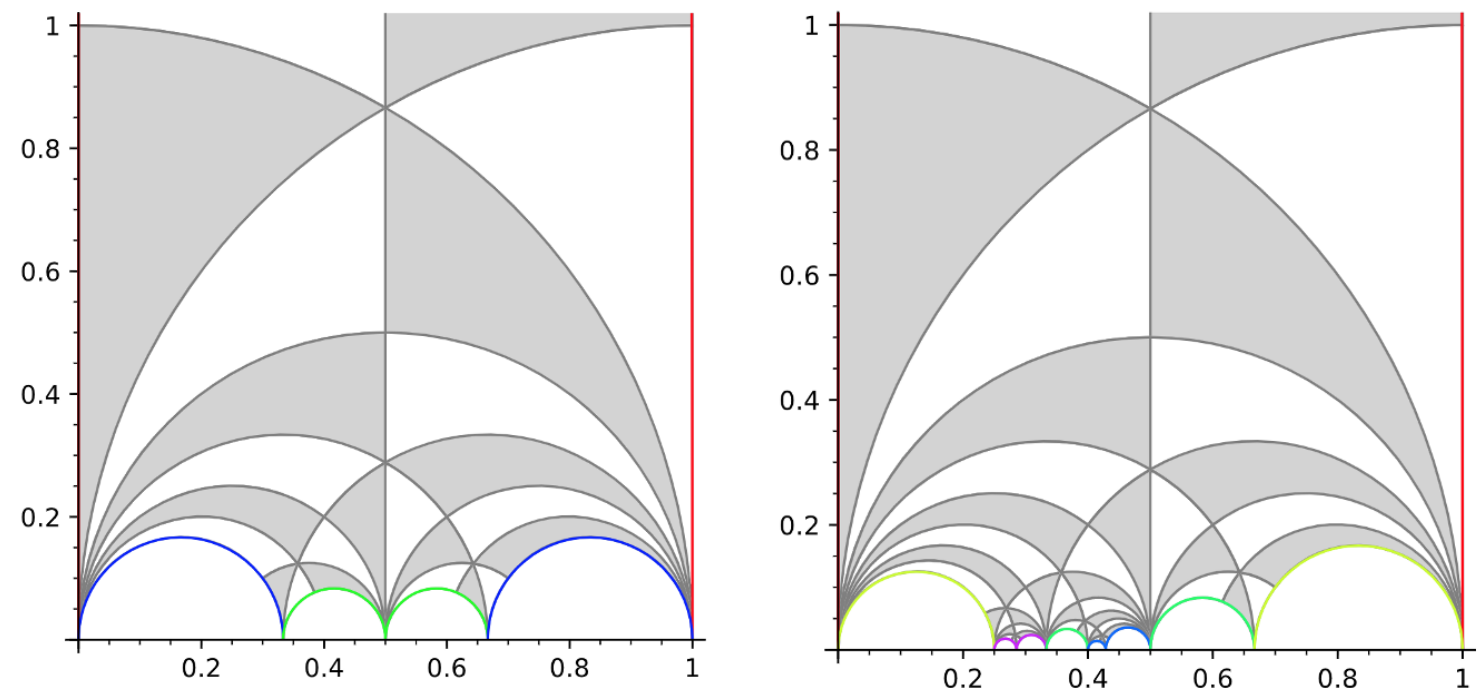

Figure 3. Triangulation of $X_{1}(6)$ and $X_{1}(7)$. Sides with equal colors are to be identified.

We note that formula (3.18) also holds with $\Gamma_{1}(n)$ replaced by any of the other subgroups, and thus reduces the computation of the degree of the fundamental line bundle of the universal surface to computing the index of a subgroup. We derive these formulas ${ }^{13}$ in appendix B. For example, the congruence subgroups $\Gamma(n)$, which are associated to elliptic curves with a pair of $n$-torsion points, have index:

$$
[\Gamma(1): \Gamma(n)]=n^{3} \prod_{p \mid n} 1-\frac{1}{p^{2}} .
$$

For $n=2,3,4,5,6$ the indices are computed to be $6,24,48,120$. With the identification of the fundamental line bundle analogous to Equation (3.18), this shows, that $\mathbb{Z}_{3} \times \mathbb{Z}_{3}$ is the maximum allowed torsion possible in rational elliptic surfaces, 3- and 4-folds. The $\mathbb{Z}_{4} \times \mathbb{Z}_{4}$ torsion extremizes $\mathrm{K} 3$ and is depicted in figure 6 of section 4.4, where we will come back to this topic. Higher order torsion points are not allowed.

\subsection{Remarks about the $\mathbb{Z}_{4}$ case}

When $n \geq 5$, the singularities predicted by the widths of the cusps correspond exactly to the fibers observed on the universal surface. This is not the case when $n=4$. We wish to briefly address this peculiarity ${ }^{14}$ in this section.

For $n>4$, the sum of the widths of the cusps is an integer multiple of the Euler characteristic of the universal surface, allowing us to deduce the degree of the fundamental line

\footnotetext{
${ }^{13}$ We will derive formulas for the indices of the congruence subgroups associated to Mordell-Weil torsion groups of the form $\mathbb{Z}_{m}$ and $\mathbb{Z}_{m} \times \mathbb{Z}_{m d}$. We do not have formulas for groups of the form $\mathbb{Z}_{m d} \times \mathbb{Z}_{n d}$ for $d>1$ and $m, n$ relatively prime, but the associated modular curves all have positive genus, and thus could not be the targets of a non-constant morphism from a Fano variety. See section 5 for more details.

${ }^{14}$ There are other reasons to believe the $\mathbb{Z}_{4}$ group is special. For example, the group structure in the special fiber of an elliptic surface with an $I_{2 k+1}^{*}$ is abstractly isomorphic to $\mathbb{Z}_{4}$, which means we can have non-semistable fibrations with 4 torsion.
} 
bundle using Equation (3.18) and to determine the minimal singularities without needing a Weierstrass model. When $n=4$, the index of the congruence subgroup is 12 , so the formula breaks down. As a result, we see a discrepancy between the singularities predicted by the widths of the cusps observed on the modular curve and the singularities on the universal surface with a 4-torsion section: the modular curve only forces $I_{4}+I_{1}+I_{1}$ on the elliptic curve, but since the discriminant of any rational elliptic surface has degree 12 , we need additional singularities to obtain a smooth elliptic surface over $\mathbb{P}^{1}$.

From the algebraic perspective, we have the equation for the universal elliptic curve:

$$
y^{2}+x y-t y=x^{3}-t x^{2},
$$

which extends to:

$$
y^{2}+t_{1}^{2} x y-t_{0} t_{1}^{2} y=x^{3}-t_{0} t_{1} x^{2} .
$$

Note that this equation has an $I_{1}^{*}$ singularity, which is the only time we see a cuspidal fiber in an elliptic fibration with a torsion point of order at least 4 . After a quadratic base change, ${ }^{15}$ we can also obtain a semistable rational surface which has a 4 -torsion point. The short Weierstrass equations for the two models are given below:

$$
\begin{aligned}
& y^{2}=x^{3}+t_{1}^{2} \frac{16 t_{0}^{2}-16 t_{0} t_{1}+t_{1}^{2}}{48} x-t_{1}^{3} \cdot \frac{64 t_{0}^{3}+120 t_{0}^{2} t_{1}-24 t_{0} t_{1}^{2}+t_{1}^{3}}{864}, \\
& y^{2}=x^{3}+\frac{16 t_{0}^{4}-16 t_{0}^{2} t_{1}^{2}+t_{1}^{4}}{48} x-\frac{64 t_{0}^{6}+120 t_{0}^{4} t_{1}^{2}-24 t_{0}^{2} t_{1}^{4}+t_{1}^{6}}{864} .
\end{aligned}
$$

We will come back to the $\mathbb{Z}_{4}$ model in section 4.3 from a more general perspective.

\section{Bounds on non-simply connected gauge groups from F-theory}

In this section we want to apply the bounds on torsion within the context of F-theory. Hence we first review F-theory and the role of Mordell-Weil torsion in its effective theory as first investigated in [54] and further explored in [52] which can be skipped by any expert in the field. The bounds on torsion, which were discussed in the sections before and proven more rigorously in section 5 can be translated into bounds on non-simply connected gauge groups in SUGRA theories obtained from F-theory. As we show, those bounds are surprising from pure 6-dimensional SUGRA arguments and their massless spectra. Finally, we also comment on bounds on smooth Calabi-Yau quotient torsors and their connection to discrete symmetries and superconformal matter.

\subsection{F-theory and the role of torsion}

This section is intended as a recap of Mordell-Weil torsion in F-theory models, based on $[52]$.

\footnotetext{
${ }^{15}$ This will be relevant in the construction of the modular curve associated to $T=\mathbb{Z}_{2} \times \mathbb{Z}_{4}$. See the end of appendix $\mathrm{C}$ for details.
} 
In F-theory, one considers an elliptically fibered threefold:

$$
\begin{aligned}
\mathcal{E} \rightarrow & Y_{n} \\
& \downarrow \pi, \\
& B_{n-1}
\end{aligned}
$$

given by a Weierstrass equation:

$$
y^{2}=x^{3}+f x+g
$$

The local axio-dilaton $\tau=C_{0}+i g_{I I B}^{-1}$ of type IIB string theory is identified with the complex modulus of each fiber and is allowed to vary over the complex base $B_{n-1}$. The singular fibers are located over the vanishing set of the discriminant:

$$
\Delta=4 f^{3}+27 g^{2} .
$$

The codimension one components of the discriminant locus

$$
\delta_{i} \subset V(\Delta),
$$

have the interpretation of generalized stacks of [p,q]-7-branes that host a local gauge algebra $\mathcal{G}_{i}$ according to their Tate-fiber type. The order of $\Delta$ can be enhanced, e.g. over the collision of components at codimension two $\delta_{i, j}=\delta_{i} \cap \delta_{j}$, which gives rise to matter multiplets that form representations $\mathbf{R}$ of the algebra $\mathcal{G}$. The matter representation can be inferred from the enhancement to the local algebra $\mathcal{G}_{i, j}$ via the decomposition [70] as:

$$
\mathbf{R}_{i, j}=\operatorname{adj}\left(\mathcal{G}_{i, j}\right) /\left(\operatorname{adj}\left(\mathcal{G}_{i}\right) \oplus \operatorname{adj}\left(\mathcal{G}_{j}\right)\right) .
$$

This construction gives a powerful tool to construct and classify a large class of consistent $D=10-2 d$ dimensional SUGRA theories via geometric methods.

The physics of F-theory compactifications on CY $d$-folds $Y_{d}$ with non-trivial MW torsion group, say $\mathrm{MW}_{\text {tor }}\left(Y_{d}\right)=\mathbb{Z}_{n}$, is understood as a global gauging of the center of the gauge algebra $\mathcal{G}_{i}$ resulting in a non-simply connected gauge group $G$ :

$$
G=\frac{\prod_{i} \mathcal{G}_{i}}{\mathbb{Z}_{n}} \times \hat{G}, \quad \pi_{1}(G)=\mathbb{Z}_{n}
$$

The quotient factor induces a constrained matter spectrum by projecting out representations that have a non-trivial central charge $q_{c e n}$. In general, the restriction to central charges can be seen explicitly via the construction of the torsion Shioda map $\Sigma$ in the resolved geometry. For any non-trivial torsion point $S_{k}$ and zero-section $Z$, the Shioda map defines a map from the elliptic curve to the Néron-Severi lattice of $Y_{d} .{ }^{16}$ As we have argued in the sections above, the mere presence of torsion points $S_{k}$, enforces the presence of singularities that need to be resolved leading to the following form of the Shioda map:

$$
\Sigma\left(S_{k}\right)=S_{k}-Z+\left(S \cdot c_{i}\right) C_{i, j}^{-1} f_{j}=S-Z+\frac{1}{n} \sum_{i} a_{j} f_{j} .
$$

\footnotetext{
${ }^{16}$ We ignore contributions from vertical divisors at this point.
} 
The fractional linear contribution of the resolution divisors $f_{i}$ above is determined by the $i$-th fibral curve $c_{i}$ in the resolved geometry, that is intersected by the torsion section $S$. The overall normalization $n$ is inherited from the inverse determinant of the Cartan matrix $C$, which is itself of the order of its center. Coming from a torsion section $S_{k}$ implies the Shioda map to be a trivial divisor in $H^{2}(Y)$ which therefore can be rewritten as

$$
\Xi\left(S_{k}\right)=S_{k}-Z=-\frac{1}{n} \sum_{i} a_{j} f_{j}
$$

and represents an $n$-torsion element in the quotient cohomology of $H^{1,1}(Y) /\left\langle\left[f_{i}\right]\right\rangle_{\mathbb{Z}}$. At the intersection $\delta_{i, j}$, the fibral curves in the resolved model generically split further $c_{i} \rightarrow c_{i, m}$ reflecting the nature of the enhanced singularity. The exact representation $\mathbf{R}_{i, j}$ and compatibility with the gauged $\mathbb{Z}_{n}$ centers can be inferred from intersections with the resolution divisors $f_{i}$ and the fractional element of the torsion Shioda map:

$$
\mu_{r}=\left(c_{i, m} \cdot f_{r}\right) \quad q_{c e n}=c_{i, m} \cdot \Xi\left(S_{k}\right) .
$$

All other weights can be obtained from adding fibral curves $c_{i}$ which also shows, that the central charge is $n$-fractional and only well-defined modulo 1 . However, since the torsion Shioda map is a trivial divisor the central charge must be integral to be consistent with the global gauge group factor. Field theoretically one can understand this, by promoting the fractional piece of the torsion Shioda map to a projector of the form $\Phi=\mathrm{e}^{2 \pi i \Xi\left(S_{k}\right)}$. This projector removes representations with the wrong (combination) of weights of the gauge group $G$. Therefore the above element defines a generator of the n-refined (co) weight lattice. ${ }^{17}$ Note that the spectrum computed above, is just the massless one and therefore comprises only a small sub-sector of the full theory. However, due to the geometric realization of the torsion Shioda map, it seems convincing that the constrained (co-) charge lattice extends to the full massive sector of the theory as well. More generally it is expected that the non-simply connected gauge groups $G$ also affect the presence of dyonic line and surface operators in the theory [71]. Similarly, we note that the presence of torsion affects the interpretation of $(p, q)$-strings as combined states of fundamental strings and $D 1$ branes. A general $(p, q)$-string (with $p$ and $q$ co-prime) that couples to the IIB $\left(B_{2}, C_{2}\right)$-fields with the respective quanta can always be rotated into the fundamental $(1,0)$ string picture by virtue of an $\mathrm{SL}(2, \mathbb{Z})$ transformation. However, under the reduced congruence subgroups we do not have the full transformation group at our disposal and therefore not every $(p, q)$ combination allows for such a transformation. As an example, for a parametrization of a $\Gamma_{1}(n)$ matrix, one may consider the following action on a fundamental string:

$$
\left(\begin{array}{cc}
1+a n & r \\
k n & 1+b n
\end{array}\right)\left(\begin{array}{l}
1 \\
0
\end{array}\right)=\left(\begin{array}{c}
1+a n \\
k n
\end{array}\right), \quad \text { with } a, b, r, k \in \mathbb{Z} .
$$

\footnotetext{
${ }^{17}$ Note that a similar effect can be resembled in the presence of a free Mordell-Weil group, that gives rise to a U(1) symmetry that can embed non trivially inside the center of other non-Abelian group factors [49, 50]. Although having a similar origin, these factors can only be present in the presence of additional Abelian symmetries, which we do not want to focus on in this work.
} 
One finds that the $D 1$ brane charge has to be divisible by the order $n$ to allow for a rotation into the fundamental string picture. This might be interpreted in two ways: either the $(p, q)$ string states with odd $q-D 1$ charge are fully absent from the spectrum, or these states can be present but come from a different sector of the theory which cannot be described as a fundamental string in any given $\Gamma_{1}(n)$ frame. However, we take the non-trivial behavior of more general string states as evidence, that torsion also affects the massive sector of the theory in a non-trivial way and would like to return to this interesting question in future work.

An $\boldsymbol{s u}(\mathbf{2}) / \mathbb{Z}_{\mathbf{2}}$ example. The simplest direct example is that of an $s u(2) / \mathbb{Z}_{2} \sim \mathrm{SO}(3)$ group. As $\mathrm{SO}(3)$ does not admit two dimensional representations, it is directly evident, that the fundamental of the $s u(2)$ covering algebra, must be absent from the spectrum. Let's now engineer this model in F-theory, via an $\mathbb{Z}_{2}$ torsion point. There, the $s u(2)$ gauge algebra is in fact forced upon us directly, as can be seen using the modular curve of $X_{1}(2)$. Explicitly, the $\Gamma_{1}(2)$ congruence subgroup is generated by upper triangle matrices modulo two. To find the generic singularity(s) we have to find the cusp(s) of the modular curve and their widths. As reviewed in section 3.3, we first have to find the triangulation of $X_{1}(2)$ by pulling back the standard triangulation of $X(1)$. The degree of this map is given in Equation (3.18) for $n=2$ which we explicitly compute to be:

$$
\left[\Gamma(1): \Gamma_{1}(2)\right]=3
$$

We can act on the fundamental domain of $X(1)$ by the coset representatives:

$$
\alpha_{1}:\left(\begin{array}{ll}
1 & 0 \\
0 & 1
\end{array}\right), \quad \alpha_{2}:\left(\begin{array}{cc}
0 & -1 \\
1 & 0
\end{array}\right), \quad \alpha_{3}:\left(\begin{array}{cc}
0 & -1 \\
1 & 1
\end{array}\right),
$$

to obtain a fundamental domain for $X_{1}(2)$. The triangulation of the modular curve into $2 \cdot 3^{18}$ sub-regions is explicitly shown in figure 4 . There are two cusps at the points:

$$
S_{\text {cusp }}^{(2)}:\left\{0^{(2)}, \infty^{(1)}\right\}
$$

with the one at the origin having width two. This is consistent with the requirement to have the appropriate gauge algebra $\mathcal{G}$ present on which the $\mathbb{Z}_{2}$ quotient can act. We can now turn to the explicit Weierstrass model, which exactly reflects what we anticipated from the modular curve:

$$
\begin{aligned}
y^{2} & =x\left(x^{2}+a_{2} x+a_{4}\right), & a_{4} & \in \mathcal{O}\left(K_{b}^{-4}\right), & a_{2} & \in \mathcal{O}\left(K_{b}^{-2}\right) . \\
f & =a_{4}-\frac{1}{3} a_{2}^{2}, & g & =\frac{1}{27} a_{2}\left(2 a_{2}^{2}-9 a_{4}\right), & \Delta & =a_{4}^{2}\left(4 a_{4}-a_{2}^{2}\right) .
\end{aligned}
$$

There we find the expected $I_{2}$ locus over $a_{4}=0$ and the residual $I_{1}$ locus. The torsion factor restricts the algebra to $s u(2) / \mathbb{Z}_{2} \sim \mathrm{SO}(3)$. Hence, geometrically we expect the enhancement from the $I_{2}$ to an $I_{3}$ locus to be forbidden, simply because of the incompatible

\footnotetext{
${ }^{18}$ The number of subregions is the number of regions in the triangulation of $X(1)$ multiplied by the degree of the map. The triangulation of $X(1)$ has 2 regions, and the degree of the map is equal to the index of the subgroup.
} 


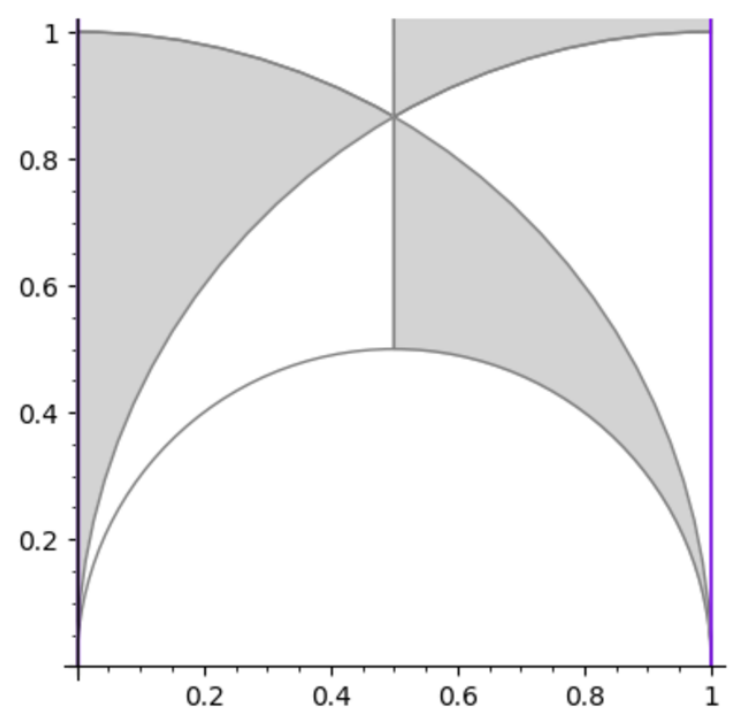

Figure 4. Triangulation of $X_{1}(2)$. A cusp point of width two is found at the origin.

center. Instead, the collision point we find is of type $I I I$, which has an associated $s u(2)$ algebra as well, and is compatible with the correct center, but which gives rise to no new matter multiplets. In the resolved geometry (e.g. see [52]) it is possible to obtain the torsion Shioda map $\Sigma\left(S_{1}\right)$ (modulo vertical divisors) as:

$$
\Sigma\left(S_{1}\right)=S_{1}-Z+\frac{1}{2} f_{1},
$$

with $f_{1}$ being the $s u(2)$ resolution divisor. By abuse of notation, we denote with $f_{1}$ also the possible $s u(2)$ weight of some representation. At codimension two, any reducible matte curve must have vanishing $(\bmod 1)$ intersection with $\Sigma\left(S_{1}\right)$. This shows, that every representation with odd $s u(2)$ Cartan charge must be absent from the spectrum.

Starting from any torsion model is a great way to systematically study and classify SUGRA theories in various dimensions with a non-trivial fundamental group. The Weierstrass model with the respective torsion point will in turn only allow for the factorization of ADE singularities that have a compatible center. This perspective is confirmed from the perspective of the congruence subgroups, that must be compatible with the local $\operatorname{SL}(2, \mathbb{Z})$ monodromy of the singularity. We have summarized the Kodaira fiber types and their congruence subgroups ${ }^{19}$ in table 1 . In order to compare a fiber monodromy matrix $M$ to be compatible with a $\Gamma_{1}(n)$ or $\Gamma(n)$ congruence subgroup, requires us to bring it into a compatible form via an $g \in \mathrm{SL}(2, \mathbb{Z})$ conjugation

$$
\widetilde{M}=g \cdot M \cdot g^{-1} .
$$

\footnotetext{
${ }^{19}$ Compatible $\Gamma_{0}(n)$ subgroups are considered in [24].
} 
In practice this can be achieved by a combination of the matrices $g_{1}=\left(\begin{array}{ll}0 & 1 \\ 1 & 1\end{array}\right)$ and the $S$ and $T$ generator. E.g. for a type $I_{2 n+1}^{*}$ fiber we can use $g=g_{1}^{2}$ to obtain

$$
\widetilde{M}_{I *_{2 n+1}}=\left(\begin{array}{c}
1+4 n-1-2 n \\
2 n+8 n-3-4 n
\end{array}\right) \in \Gamma_{1}(4) \in \Gamma_{1}(2) .
$$

For the monodromies of type $I_{n}, I I I, I V^{*}$ and $I I I^{*}$ fibers, conjugation by $g=S \cdot g_{1}$. For type $I V$ and $I_{2 n}^{*}$ one can conjugation by $g=S \cdot T \cdot S$ and $g=g_{1}$ respectively to achieve the right forms. Apart from the $I_{n}$ fibers, we find a perfect match between the centers of the covering algebra and their associated congruence subgroup. In the $s u(n)$ case we also find compatibility to $\Gamma(n)$ groups, that one expects to encode $\mathbb{Z}_{n} \times \mathbb{Z}_{n}$ centers. More generally, there might be the possibility, for some torsion to only mod out a subcenter of some $s u(n)$ algebra, when some $\mathbb{Z}_{n \cdot m}$ factor is present. The embedding of the torsion inside the $s u(n)$ center is hardly visible from the monodromy picture but in the resolved geometry, which we will comment on in section 4.3. When considering larger torsion, the options of compatible ADE fibers get sparser but are still unbounded in principle, thanks to the $s u(n)$ factors. In the next section, we want to switch gears and investigate whether 6 dimensional anomalies can constrain the order $n$ in a meaningful way.

\subsection{D SUGRA with large putative torsion}

From a field theory perspective, there is no direct reason why torsion of higher orders should not exist, given that there is always some $s u(n)$ covering algebra with the given center that can satisfy the strong 6d SUGRA anomalies. These constraints are given as:

$$
\begin{aligned}
H-V+29 T=273, \quad 9-T & =a \cdot a, & & \text { (pure-gravitational) } \\
-\frac{1}{6}\left(A_{\mathrm{adj}_{\kappa}}-\sum_{\mathbf{R}} x_{\mathbf{R}} A_{\mathbf{R}}\right) & =a \cdot b_{\kappa}, & & \text { (Non-Abelian-gravitational) } \\
B_{\mathrm{adj}_{\kappa}}-\sum_{\mathbf{R}} x_{\mathbf{R}} B_{\mathbf{R}} & =0, & & \text { (Pure non-Abelian) } \\
\frac{1}{3}\left(\sum_{\mathbf{R}} x_{\mathbf{R}} C_{\mathbf{R}}-C_{\mathrm{adj}_{\kappa}}\right) & =b_{\kappa}^{2}, & &
\end{aligned}
$$

with $H, V$ and $T$ being the number of massless hyper-, vector-and tensor multiplets in the theory and $x_{\mathbf{R}}$ the number of hypermultiplets in representations $\mathbf{R}$. The anomaly coefficients $a$ and $b_{\kappa}$ transform as $\mathrm{SO}(1, T)$ vectors and the anomalies can be evaluated using the following group theory coefficients: ${ }^{20}$

\begin{tabular}{|c|c|c|c|c|c|}
\hline Representation & Dimension & $A_{\mathbf{R}}$ & $B_{\mathbf{R}}$ & $C_{\mathbf{R}}$ & $E_{\mathbf{R}}$ \\
\hline Fundamental & $n$ & 1 & 1 & 0 & 1 \\
\hline Adjoint & $n^{2}-1$ & $2 n$ & $2 n$ & 6 & 0 \\
\hline
\end{tabular}

\footnotetext{
${ }^{20}$ We do not consider su(2) and su(3) groups that have different $C$ and vanishing $B$ and $E$ type coefficients.
} 


\begin{tabular}{|c|c|c|c|c|c|}
\hline Fiber & $(f, g, \Delta)$ & Monodromy & Subgroups & algebra & center \\
\hline$I I^{*}$ & $(\geq 1,1,2)$ & $\left(\begin{array}{cc}1 & 1 \\
-1 & 0\end{array}\right)$ & - & - & - \\
\hline$I_{n}$ & $(0,0, n)$ & $\left(\begin{array}{ll}1 & n \\
0 & 1\end{array}\right)$ & $\Gamma_{1}(n), \Gamma(n)$ & $s u(n)$ & $\mathbb{Z}_{n}$ \\
\hline$I I I$ & $(\geq 1, \geq 2,3)$ & $\left(\begin{array}{cc}0 & -1 \\
1 & 0\end{array}\right)$ & $\Gamma_{1}(2)$ & $s u(2)$ & $\mathbb{Z}_{2}$ \\
\hline$I V$ & $(\geq 2,2,4)$ & $\left(\begin{array}{cc}0 & 1 \\
-1 & -1\end{array}\right)$ & $\Gamma_{1}(3)$ & $s u(3)$ & $\mathbb{Z}_{3}$ \\
\hline$I_{2 n}^{*}$ & $(\geq 2,3,6+2 n)$ & $\left(\begin{array}{cc}-1 & -2 n \\
0 & -1\end{array}\right)$ & $\Gamma_{1}(2), \Gamma(2)$ & $s o(8+4 n)$ & $\mathbb{Z}_{2} \times \mathbb{Z}_{2}$ \\
\hline$I_{2 n+1}^{*}$ & $(\geq 2,3,7+2 n)$ & $\left(\begin{array}{cc}-1 & -2 n-1 \\
0 & -1\end{array}\right)$ & $\Gamma_{1}(2), \Gamma_{1}(4)$ & $s o(10+4 n)$ & $\mathbb{Z}_{4}$ \\
\hline$I V^{*}$ & $(\geq 3,4,8)$ & $\left(\begin{array}{cc}-1 & -1 \\
1 & 0\end{array}\right)$ & $\Gamma_{1}(3)$ & $E_{6}$ & $\mathbb{Z}_{3}$ \\
\hline$I I I^{*}$ & $(3, \geq 5,9)$ & $\left(\begin{array}{cc}0 & -1 \\
1 & 0\end{array}\right)$ & $\Gamma_{1}(2)$ & $E_{7}$ & $\mathbb{Z}_{2}$ \\
\hline$I I^{*}$ & $(4, \geq 5,10)$ & $\left(\begin{array}{cc}0 & -1 \\
1 & 1\end{array}\right)$ & - & $E_{8}$ & - \\
\hline
\end{tabular}

Table 1. Summary of ADE fiber types, their Weierstrass singularity and associated $s l(2, \mathbb{Z})$ monodromy. Column four shows compatible $\Gamma_{1}(n)$ and $\Gamma(n)$ subgroups matching the center of the associated algebra. $I_{n}$ monodromies are both compatible with $\mathbb{Z}_{n}$ and $\mathbb{Z}_{n} \times \mathbb{Z}_{n}$ torsion points.

To match the F-theory geometry we make the following identification of base divisors $a \sim K_{b}$ and $b_{\kappa} \sim \mathcal{Z}_{\kappa}$ of $s u(n)_{\kappa}$ whereas $\cdot$ denotes the intersection in the base.

When considering $s u(n) / \mathbb{Z}_{n}$ groups, there are several massless representations that can be exclude straight away due to their incompatible $\mathbb{Z}_{n}$ central charges. These include fundamentals and (single times) antisymmetric representations as those are associated to $s u(n) \rightarrow s u(n+1)$ and $s u(n) \rightarrow s o(2 n)$ algebra enhancements at codimension two that have incompatible centers for large enough $n .^{21}$ Let's exclude anti-symmetric matter directly and reformulate the anomalies as the following conditions on fundamental and adjoint hypermultiplets:

$$
x_{\text {fund }}=2 n\left(x_{\mathrm{adj}}-1\right), \quad x_{\mathrm{adj}}=g=1+\frac{1}{2}\left(\mathcal{Z}^{2}-\mathcal{Z} \cdot K_{b}^{-1}\right),
$$

where $g$ is the genus of the divisor $\mathcal{Z}$ in the base. Let us consider solutions compatible with a putative $s u(n) / \mathbb{Z}_{n}$ group over the divisor $\mathcal{Z}$. A first solution is that of theory, with

\footnotetext{
${ }^{21}$ The later ones have at most an $\mathbb{Z}_{2}, \mathbb{Z}_{2} \times \mathbb{Z}_{2}$ or $\mathbb{Z}_{4}$.
} 
only adjoint hyper multiplets, realized by a genus one curve of self-intersection zero. This leaves only the pure gravitational anomaly that can be solved, given the following amount of pure neutral hypermultiplets:

$$
H_{\text {neutral }}=272+n-29 T \text {. }
$$

Let's try to engineer that model more concretely in F-theory. As a base we choose $d P_{9}$ and the $s u(n)$ divisor to be $z=0: \mathcal{Z} \in K_{b}^{-1}$ to guarantee it to be of genus $g=1$. The factorization of a Tate model

$$
y^{2}+x^{3}+a_{1} y x+a_{2} x^{2}+a_{3} y+a_{4} x+a_{6}, \quad\left[a_{i}\right] \in K_{b}^{-i},
$$

is given as:

$$
a_{2} \rightarrow b_{1} z \quad a_{3} \rightarrow c_{0} z^{3}, \quad a_{4} \rightarrow d_{0} z^{4}, \quad a_{6} \rightarrow e_{0} z^{6},
$$

with the $c_{0}, d_{0}, e_{0}$ being generic non-vanishing constants. The above factorization gives an $s u(6)$ over $z=0$, without any further codimension two loci since $\left(K_{b}^{-1}\right)^{2}=0$. By further setting $e_{0}$ and $c_{0}$ to zero, this singularity can be enhanced to $s u(7)$ and $s u(8)$ respectively or by further setting $b_{1}=0$ increasing the singularity to $s u(9)$. This spectrum admits only a single adjoint representation in its massless spectrum and looks naively like a possible $s u(n) / \mathbb{Z}_{n}$ model for values $n>4$. Especially the later one admits a torsion point of order three as it is a variant of the Schoen $[40,63]$ but not of order nine as the massless spectrum might suggest.

A second solution to the anomaly constraints above is to put the $s u(n)$ factor over a $\mathbb{P}^{1}$ of self-intersection -2 in the base, hosting no adjoint hypermultiplets but instead $2 \mathrm{n}$-fundamentals. In order to get that spectrum consistent with the enhancement rules we introduce another $s u(n)$ gauge factor, over another -2 curve giving rise to the enhancement:

$$
s u(n)_{1} \cap s u(n)_{2} \rightarrow s u(2 n),
$$

consistent with the $\mathbb{Z}_{n}$ factor. This configuration can be continued with up to $\mathrm{k} s u(n)$ factors, intersecting each other as:

$$
-s u(n)_{1}-s u(n)_{2}-\ldots s u(n)_{k}-
$$

forming the structure of an affine $\mathrm{su}(\mathrm{k})$ Dynkin diagram in the base. The resulting gauge group is of $s u(n)^{k}$ type with $\mathrm{k}$, bi-fundamental representations of type $\left(\mathbf{1}, \mathbf{N}_{i}, \overline{\mathbf{N}}_{i+1}, \ldots, \mathbf{1}\right)$ consistent with a naive $\mathbb{Z}_{n}$ factor. Such a configuration can cancel the gravitational anomaly given the existence of

$$
H_{\text {neutral }}=273-k-29 T,
$$

neutral hyper and tensor multiplets present.

All the above models have a massless spectrum which is seemingly consistent with an $s u(n) / \mathbb{Z}_{n}$ gauge group or multiple copies of those. It seems here that the pure gauge theory anomalies do not give much guidance, on what bounds on the fundamental group we might expect. However, we not, that we mainly argued with the massless sub-sector of 
the theory and there is a priori no reason to assume, that massive modes must respect the putative $s u(n) / \mathbb{Z}_{n}$ group as well. It is arguably more convincing, that the massive modes respect the (co-)charge refinement that is explicitly enforced by the torsion Shioda map and the reduced monodromy of $\Gamma_{1}(n)$, that also acts non-trivial on $(p, q)$-strings as argued in section 4.1. Similarly, it was shown in the IIA dual geometry [54] that torsion restricts Ramon-Ramon charges of BPS solitons resulting in a non-simply connected group in the same way as in F-theory.

\subsection{Higher order and non-prime torsion}

In this section we want to comment on torsion points and their field theory counterparts in the case, when the torsion affects only a sub-center of the gauge algebra as well when it is non-prime and how this is realized in the geometry itself.

Let's consider e.g. the case when only a $\mathbb{Z}_{m}$ sub-factor of some $s u(n \cdot m)$ algebra becomes gauged. This is visible e.g. in the $\mathbb{Z}_{2}$ torsion model by further specializing $a_{4} \rightarrow b_{2}^{2}$, which enhances the $s u(2)$ to an $s u(4)$. The spectrum however is still constraint to an $s u(4) / \mathbb{Z}_{2} \sim \mathrm{SO}(6)$ sub-factor, consistent with the existence of antisymmetric 6-plet states. This is also consistent with the $\mathbb{Z}_{2}$ symmetry among 0 -and $\mathbb{Z}_{2}$ torsion which dictates the torsion section to intersect the second $s u(4)$ resolution divisor, leaving a torsion Shioda map of the form:

$$
\Sigma\left(S_{1}\right)=S_{1}-Z+\frac{1}{2}\left(f_{1}+2 f_{2}+f_{3}\right)
$$

The factor $1 / 2$ shows, that the torsion Shioda map is indeed of order two, which gauges the $\mathbb{Z}_{2}$ sub-center of the $s u(4)$ algebra. This does not allow for 4 -plets with weight $(1,0,0)$ but the anti-symmetric 6 -plets with $(0,1,0)$ weight, which can be seen in the associated Weierstrass model explicitly [52]. It is easy to extend this observation by noting, that globally, there is the freedom to redefine the zero-and order k-torsion section. This symmetry on the other hand, must extend to the appearing gauge algebra factors and hence a cyclic automorphism of the affine Dynkin diagram itself [50]. Indeed, this can be geometrically seen by noting that each order $k$ - section can intersect an $s u(n \cdot k)$ at the $\mathrm{k}$-th-node. The inverse Cartan matrix of the $s u(n \cdot k)$ then comes with a factor $\frac{1}{\operatorname{det}(G)}=\frac{1}{n k}$. However, the $k$-th. row of $C^{-1}$ comes with a k-multiple and hence, the overall contribution of the $s u(n k)$ is only $n$ - fractional. Hence the torsion Shioda map $\Sigma\left(S_{k}\right)$, effects only a sub-center.

Starting from the torsion forms, it is interesting to observe that, with order $M W(Y)_{\text {tor }}=\mathbb{Z}_{n}$ for $n>3$, several additional singularities beyond the expected minimal $s u(n)$ start appearing. To consider this effect in more detail, we go back to the $\mathbb{Z}_{4}$ example with general Weierstrass model:

$$
\begin{array}{ll}
y^{2}+b_{1} x y+b_{1} b_{2} y=x^{3}+b_{2} x^{2}, & {\left[b_{1}\right] \in K_{b}^{-1}, \quad\left[b_{2}\right] \in K_{b}^{-2},} \\
f_{4}=-\frac{1}{48} b_{1}^{4}+\frac{1}{3} b_{1}^{2} b_{2}-\frac{1}{3} b_{2}^{2},, & g_{4}=-\frac{1}{864}\left(b_{1}^{2}-8 b_{2}\right)\left(b_{1}^{4}-16 b_{1}^{2} b_{2}-8 b_{2}^{2}\right), \\
\Delta=-\frac{1}{16} b_{1}^{2} b_{2}^{4}\left(b_{1}^{2}-16 b_{2}\right) . &
\end{array}
$$

The above geometry suggests a minimal $(s u(2) \times s u(4)) / \mathbb{Z}_{4}$ gauge group. The presence of the $s u(2)$ factor is especially surprising, as it does not appear in the $\Gamma_{1}(4)$ modular curve, 

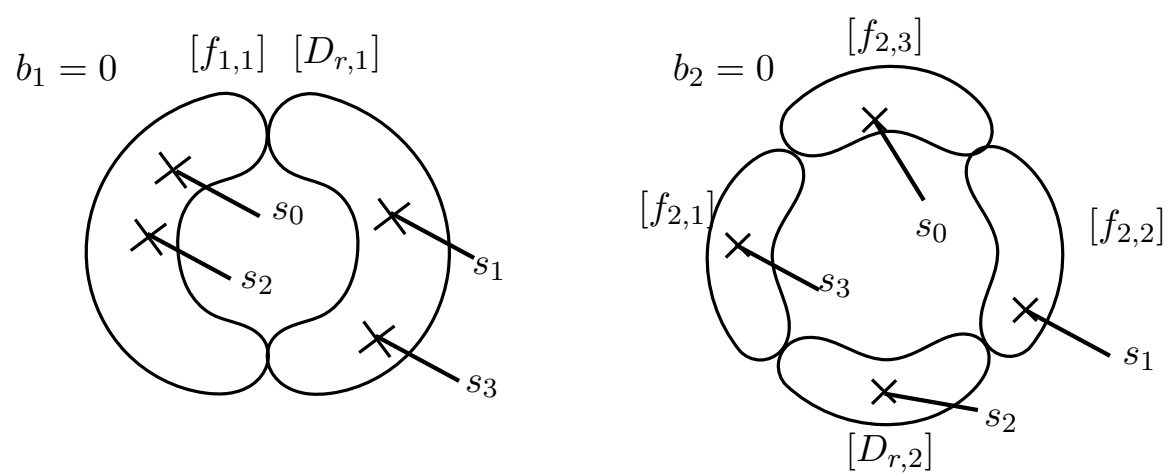

Figure 5. Depiction of the generic resolved $\mathbb{Z}_{4}$ torsion model and its intersection with the torsion sections $s_{i}$. The intersection pattern with the resolution divisors is compatible with the cyclic order four movement for the $s u(4)$ fibers and an order two subgroup for $s u(2)$ fibers.

which we discussed in section 3.4. From the argument above, it seems also puzzling how the torsion section can consistently intersect the local $s u(2)$, although being of order 4 . Therefore we will analyze the resolved model in more detail in the following, based on the codimension two resolution with PALP id $(3415,0)[28,58]$ and equation:

$$
\begin{aligned}
& p_{1}=f_{2,1} f_{1,1} f_{2,2} f_{2,3}^{2} z_{0}^{2}+b_{1} f_{2,1} f_{2,2} f_{2,3} z_{1} z_{3}+b_{2} f_{1,1} z_{2}^{2}, \\
& p_{2}=f_{2,2} z_{1}^{2}+f_{2,1} z_{3}^{2}+f_{1,1} z_{0} z_{2},
\end{aligned}
$$

with $z_{i}$ toric sections and $f_{i}$ some resolution divisors that have Stanley-Reisner ideal:

$$
\begin{aligned}
S R I:\{ & f_{2,2} z_{2}, z_{0} z_{2}, z_{1} z_{2}, f_{2,1} z_{2}, f_{2,3} z_{2}, z_{3} z_{2}, z_{0} z_{1}, f_{1,1} z_{1}, f_{2,3} z_{1}, z_{1} z_{3}, \\
& \left.f_{2,2} z_{3}, z_{0} z_{3}, f_{1,1} z_{3}, f_{2,3} z_{3}, f_{2,2} z_{0}, f_{1,1} f_{2,2}, f_{1,1} f_{2,3}, f_{2,1} z_{0}, f_{2,1} f_{1,1}\right\} .
\end{aligned}
$$

The resolved fibers are shown in figure 5 together with the intersection of the four torsion sections $\left\{z_{0}, z_{1}, z_{2}, z_{3}\right\}$ which we call $s_{i}$ denoted by their order $i$ in the MW group law [50]. It is worth comparing the intersection structure of the torsion sections withing the affine $s u(2)$ and $s u(4)$ fiber. For the later one we observe how each torsion section $s_{i}$ intersects exactly the $i$-th node in the fiber while for the $s u(2)$ they intersect the same node in even/odd pairs. E.g. the zero-section and the $s_{2}$ section intersect the affine node, whereas the second fibral curve is intersected by the odd sections $s_{1}$ and $s_{3}$. This structure might suggest the enforced presence of the $s u(2)$ sub algebra as it is predicted for a $X_{1}(2)$ modular curve, due to the non-trivial $\mathbb{Z}_{2}$ subgroup of $\mathbb{Z}_{4}$. This can also be observed via the two different torsion Shioda maps $\Sigma\left(S_{i}\right)$, that give a trivial $i$-torsional divisor in the $N S$ lattice via the expressions:

$$
\begin{aligned}
& \Sigma\left(S_{1}\right)=\left[s_{1}\right]-\left[s_{0}\right]-\frac{1}{2} f_{1,1}+\frac{1}{4}\left(f_{2,1}+2 f_{r}+3 f_{2,2}\right), \\
& \Sigma\left(S_{2}\right)=\left[s_{2}\right]-\left[s_{0}\right]+\frac{1}{2}\left(f_{2,1}+2 f_{r}+f_{2,2}\right)
\end{aligned}
$$




\begin{tabular}{|c|c|c|}
\hline Gauge group: & $\left(s u(4)^{2} \times s u(2)\right) / \mathbb{Z}_{4} \times U(1)$ & $(s u(6) \times s u(3) \times s u(2)) / \mathbb{Z}_{6}$ \\
\hline$\left(h^{1,1}, h^{2,1}\right)$ & $(19,19)$ & $(19,19)$ \\
\hline$H:$ & $2 \times \mathbf{1 5} \oplus \mathbf{3} \oplus 20 \times \mathbf{1}$ & $\mathbf{3 5} \oplus \mathbf{8} \oplus \mathbf{3} \oplus 20 \times \mathbf{1}$ \\
\hline$T:$ & 9 & 9 \\
\hline
\end{tabular}

Table 2. Summary of massless $6 \mathrm{D}$ F-theory spectra with $\mathbb{Z}_{4}$ and $\mathbb{Z}_{6}$ fundamental group, geometrically realized by the Schoen manifold.

with $f_{r}=-\left(f_{2,2}+f_{2,3}+f_{2,1}\right)$. We observe the first Shioda map gives a 4 -torsion element whereas the second one is only 2-torsion and only sees the $\mathbb{Z}_{2}$ subgroup of $s u(4)$ but not the $s u(2)$ at all. The above model can be completed to a 6D SUGRA model, which requires the absence of non-flat fibers, or $(4,6,12)$ points, that are generically present over $b_{1}=b_{2}=0$ and hence requires $\left(K_{b}^{-1}\right)^{2}=0$ which can be achieved over a rational elliptic surface as the base. In order to make the model simple, we can factor $a_{2} \rightarrow c_{1} b_{1}$, giving rise to two copies of $s u(4)$ factors discussed above. This model can also be arranged as a variant of the Schoen manifold [40], which, in addition to the $\left(s u(4)^{2} \times s u(2)\right) / \mathbb{Z}_{4}$ admits a non-higgsable $\mathrm{U}(1)$ factor. The full matter spectrum of the model is specified in table 2 and can be shown to satisfy gauge and gravitational anomalies The observations above, are pathological for non-prime $\mathbb{Z}_{n}$ order torsional models. The presence of $s u(m)$ gauge factors, where $m$ are divisors of $n$ forces $(4,6,12)$ points over generic Fano bases that can be avoided over $\mathrm{dP}_{9}$. The maximal cases possible are $\mathbb{Z}_{6}$ for a single factor and $\mathbb{Z}_{3} \times \mathbb{Z}_{3}$ for two factors, as concretely constructed in [54]. The Weierstrass coefficients of the former one is given as

$$
\begin{aligned}
y^{2}+a_{1} x y & +\frac{1}{32}\left(a_{1}-b_{1}\right)\left(3 a_{1}+b_{1}\right)\left(a_{1}+b_{1}\right)=x^{3}+\frac{1}{8}\left(a_{1}-b_{1}\right)\left(a_{1}+b_{1}\right) x^{2} \\
f & =\frac{1}{192} b_{1}\left(3 a_{1}^{3}-3 a_{1}^{2} b_{1}-3 a_{1} b_{1}^{2}-b_{1}^{3}\right) \\
g & =\frac{1}{110592}\left(3 a_{1}^{2}-6 a_{1} b_{1}-b_{1}^{2}\right)\left(9 a_{1}^{4}-6 a_{1}^{2} b_{1}^{2}-24 a_{1}^{3} b_{1}-11 b_{1}^{4}\right) \\
\Delta & =\frac{1}{2^{24}}\left(a_{1}-5 b_{1}\right)\left(3 a_{1}+b_{1}\right)^{2}\left(a_{1}+b_{1}\right)^{3}\left(a_{1}-b_{1}\right)^{6}, \quad a_{1}, b_{1} \in \mathcal{O}\left(K_{b}^{-1}\right),
\end{aligned}
$$

and is also naturally described by a Schoen manifold, in order to keep all $(4,6,12)$ points absent. Note that the generic $I_{6}, I_{3}$ and $I_{2}$ singular fibers expected from the cusp points of the modular curve, as discussed in section 3.3 and depicted in figure 3 are present. This model gives rise to a good F-theory vacuum in six dimensions with $(s u(6) \times s u(3) \times s u(2)) / \mathbb{Z}_{6}$ gauge group, whose massless spectrum is summarized in table 2 .

The modular curve perspective gives a good understanding of which minimal singularities are to be expected in certain torsion models. Let's go to a higher $n$-torsion point say $\mathbb{Z}_{8}$ e.g. over a $\mathbb{F}_{0} \sim \mathbb{P}_{\left[t_{0}, t_{1}\right]}^{1} \times \mathbb{P}_{\left[s_{0}, s_{1}\right]}^{1}$ base which we derive explicitly in appendix $A$ and analyze the associated Weierstrass model directly. We repeat the model here, as:

$$
\begin{aligned}
y^{2}+ & \left(s_{0} s_{1} t_{0} t_{1}+\left(s_{0} t_{0}-s_{1} t_{1}\right)\left(2 s_{1} t_{1}-s_{0} t_{0}\right)\right) x y+s_{0} s_{1}^{3} t_{0} t_{1}^{3}\left(s_{0} t_{0}-2 s_{1} t_{1}\right)\left(s_{1} t_{1}-s_{0} t_{0}\right) y \\
& =x^{3}+s_{1}^{2} t_{1}^{2}\left(s_{0} t_{0}-2 s_{1} t_{1}\right)\left(s_{1} t_{1}-s_{0} t_{0}\right) x^{2}
\end{aligned}
$$




\begin{tabular}{|c|c|}
\hline $\mathrm{MW}_{\text {tor }}$ & Fibers \\
\hline $\mathbb{Z}_{7}$ & $3 I_{7}$ \\
\hline $\mathbb{Z}_{8}$ & $2 I_{8}+I_{4}+I_{2}$ \\
\hline $\mathbb{Z}_{6} \times \mathbb{Z}_{2}$ & $3 I_{6}+3 I_{2}$ \\
\hline $\mathbb{Z}_{4} \times \mathbb{Z}_{4}$ & $6 I_{4}$ \\
\hline
\end{tabular}

Table 3. Summary of large torsion models, that cannot be realized in 3- and 4-folds and their singular fibers. All of them are extremal K3's.

with discriminant:

$$
\Delta=\frac{-1}{16} s_{0}^{2} s_{1}^{8} t_{0}^{2} t_{1}^{8}\left(s_{0} t_{0}-2 s_{1} t_{1}\right)^{4}\left(s_{0} t_{0}-s_{1} t_{1}\right)^{8}\left(s_{0}^{2} t_{0}^{2}-8 s_{0} s_{1} t_{0} t_{1}+8 s_{1}^{2} t_{1}^{2}\right) .
$$

This model admits two $I_{2}$, one $I_{4}$ and three $I_{8}$ fibers as well as two $(8,12,24)$ codimension two singularities. Therefore, the $\left(s u(8)^{3} \times s u(4) \times s u(2)^{2}\right) / \mathbb{Z}_{8}$ six dimensional SUGRA model cannot exist. In the next section we show, however that a related model in eight dimensions can exist.

\subsection{K3: 8-dimensional exceptions}

The only way to avoid the $(8,12)$ singularities at codimension two is by restricting the base to be one-dimensional, that is a K3 surface. This allows some additional possible higher order torsion models to exist exclusively as 2-folds, as opposed to 3- and 4-folds. Hence their underlying 8D SUGRA theories, obtained from F-theory comprise of isolated theories as we discuss in the following. The $\mathbb{Z}_{8}$ model is one of the few exceptional torsion models, only ${ }^{22}$ present in elliptic K3's. The groups and their fiber configurations are summarized in table 3, that is adapted from a table in [32]. As can be found in table 3, all elliptic K3's are of extremal type, which means that their Néron-Severi group has maximal possible rank 20. In short, no elements of $h^{1,1}(Y)$ can contribute to complex structure deformations which results in a rigid geometry. In fact, the $T=\mathbb{Z}_{8}$ threefold given in (4.35) can be viewed as an enforced K3-fibration over a base $\mathbb{P}^{1}$ in terms of $\left[t_{0}, t_{1}\right]$ coordinates that preserves the torsion. Deleting the $\mathbb{P}^{1}$ by effectively setting the coordinates to one, gives the consistent K3 with the correct fibers.

In this regard, also their associated 8D F-theory vacua are special. Their rigidity, does not allow for a minimal SUSY preserving compactification to lower dimension ${ }^{23}$ compatible with the torsion. Moreover, the rigidity, also does not allow for a stable degeneration limit at finite distance in the moduli space into two rational elliptic surfaces, as those require at least one free complex structure modulus to assign the proper scaling. Therefore, these models do not have a (geometric) ${ }^{24}$ heterotic dual either. This is consistent with the fact, that the groups do not fit into $E_{8} \times E_{8}$, which is also reflected by the fact, that none of the torsion factors, are discrete finite subgroups of $E_{8}$.

\footnotetext{
${ }^{22}$ As opposed to Calabi-Yau fibrations of higher dimension.

${ }^{23}$ Note that trivial, fibrations over a torus or K3 [53] are still possible.

${ }^{24}$ In [75] non-geometric heterotic-F-theory dual models in eight and lower dimensions have been proposed,
} 

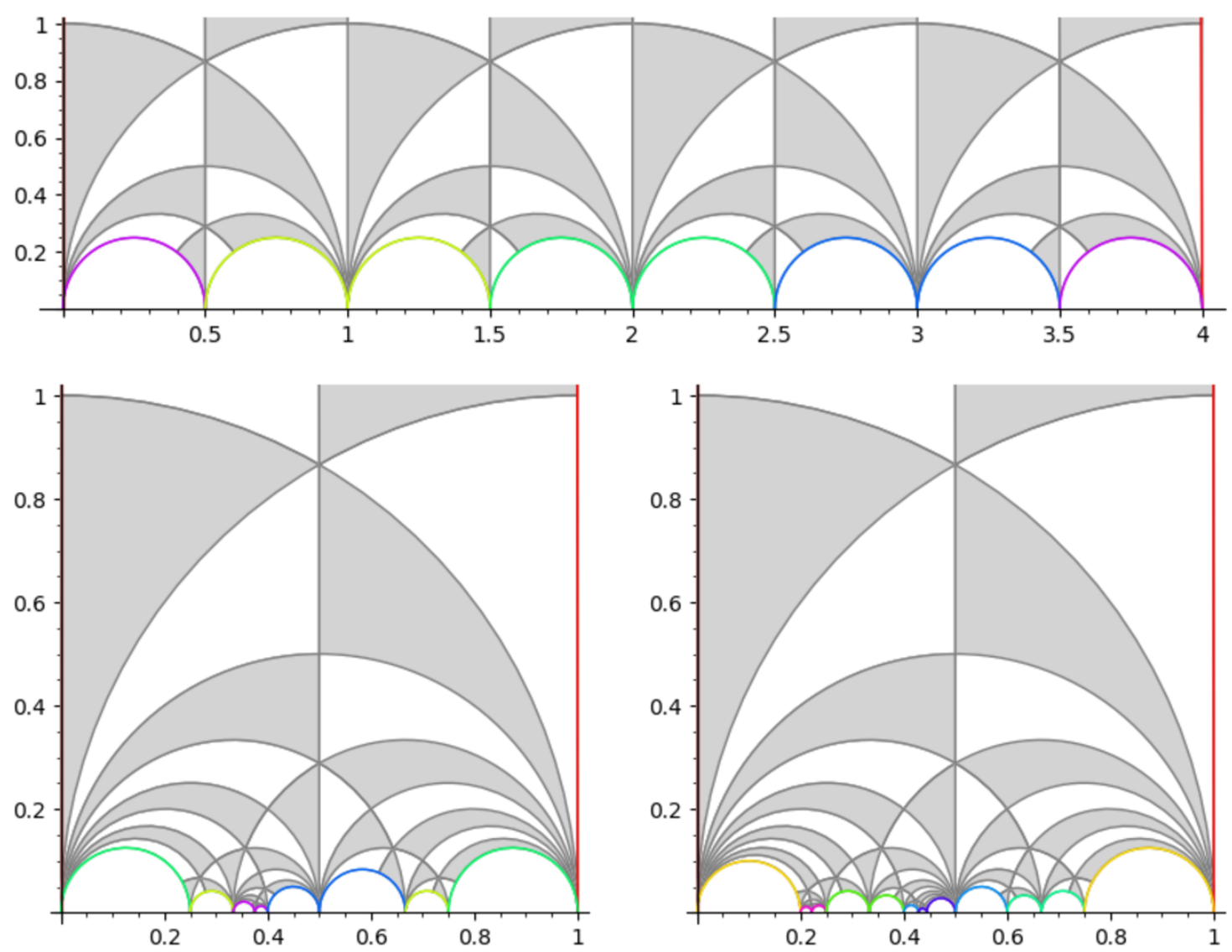

Figure 6. Triangulations of $X(4), X_{1}(8)$ and $X_{1}(9)$. Colors highlight identified lines, allowing to read off the cusp points and their widths.

The same conclusions can be obtained, by simply considering the modular curves of the higher torsion models which naturally explains the absence of even larger torsion in K3. In figure 6 we depicted the three modular curves that correspond to $\mathbb{Z}_{4} \times \mathbb{Z}_{4}, \mathbb{Z}_{8}$ and $\mathbb{Z}_{9}$ torsion model explicitly. The $\mathbb{Z}_{4} \times \mathbb{Z}_{4}$ modular curve, admits six cusp points at

$$
S_{\text {cusp }}^{(4 \times 4)}:\left\{0^{(4)}, 1 / 2^{(4)}, 1^{(4)}, 2^{(4)}, 3^{(4)}, \infty^{(4)}\right\},
$$

where we highlighted their widths as superscripts. The associated fibers are consistent with the expectation from the K3 classification. Similarly, the $\mathbb{Z}_{8}$ curve, discussed earlier admits six cusps

$$
S_{\text {cusp }}^{(8)}:\left\{0^{(8)}, 1 / 4^{(2)}, 1 / 3^{(8)}, 3 / 8^{(1)}, 1 / 2^{(4)}, \infty^{(1)}\right\},
$$

consistent with K3 construction as well. Finally, we depicted also the $\mathbb{Z}_{9}$ case in figure 6 . There are eight cusps in total at

$$
S_{\text {cusp }}^{(9)}:\left\{0^{(9)}, 2 / 9^{(1)}, 1 / 4^{(9)}, 1 / 3^{(3)}, 4 / 9^{(1)}, 1 / 2^{(9)}, 2 / 3^{(3)}, \infty^{(1)}\right\} .
$$

some of them also admitting non-crepant singularities. 
Collecting all singular fibers, we end up with three $I_{9}$ and two $I_{3}$ fibers in total. Resolving these singularities while adding the class of the generic fiber and base required 30 independent Kähler parameters. This clearly over shots the maximum of 20 that K3 has at its disposal by large. With view on F-theory this also implies the absence of eight-dimensional SUGRA theories with a non-simply connected gauge group $G$ with $\pi_{1}(G)=\mathbb{Z}_{n} \times \mathbb{Z}_{m}$ beyond the bound $(n=1, m \leq 8)$ and $(n \leq 4, m \leq 4)$.

\subsection{Bounds on Calabi-Yau quotient torsors}

These results also reveal constraints on a certain class of non-simply connected, genus-one fibered Calabi-Yau 3-folds $\widehat{Y}_{3}$ which are relevant for heterotic [22, 44, 45] and F-theory compactifications $[19,21]$. The construction of the 3 -folds is described in detail in [22, 40]. We start with a smooth elliptic 3 -fold $X_{3} \rightarrow B_{2}$, and assume that we have the following extra structure:

- An automorphism $\alpha$ of $B_{2}$ which is of finite order, and which is compatible with the fibration structure.

- A non-identity element $P \in M W(Y / B)$ satisfying:

- $P$ is fixed by $\sigma$, and $n P=0$ in MW.

- $P$ is not fixed by $\sigma$, and the sum of the elements in the $\alpha$ orbit of $P$ is 0 .

We then combine the automorphism of the base with a translation by $P$ along the fibers to obtain a finite order, fixed point-free automorphism of $Y_{3}$, that we denote $\tilde{\alpha_{P}}$. We can take a simultaneous quotient of $Y_{3}$ and $B_{2}$ by the action of $\left\langle\tilde{\alpha_{P}}\right\rangle$ and $\langle\alpha\rangle$, respectively, we obtain a new fibration $Y_{3} /\left\langle\tilde{\alpha_{P}}\right\rangle \rightarrow B_{2} /\langle\alpha\rangle$.

The resulting quotient geometries have two central properties that we want to comment on.

1. Although $Y_{3} / /\left\langle\tilde{\alpha_{P}}\right\rangle$ is itself smooth, $B_{2} /\langle\alpha\rangle$ has isolated singularities over the fixed points of $\alpha$ action. The fibration has multiple fibers ${ }^{25}$ over these singular points [56].

2. Second, the new fibration no longer has a global section. This is immediate from the fact that we have multiple fibers over the singularities in the base. The zero section of the original fibration becomes a multisection of degree $n$ in the quotient fibration.

In the physics of F-theory, the $n$-multisections have been shown to lead to discrete $\mathbb{Z}_{n}$ gauge symmetries in the 6D SUGRA theory. The fixed points on the other hand carries superconformal matter theories coupled to gravity. In [19,21] consistency of such theories for all $\mathbb{Z}_{n}$ quotients have been shown.

The bounds on the MW torsion groups therefore also constrains the possibilities for this construction using torsion points. In the physics of F-theory, these constraints can be translated into bounds on discrete symmetries and gaugings of superconformal matter.

\footnotetext{
${ }^{25}$ In the context of F-theory, these fibers also appeared in the duality to the CHL string [37] and classification of little string theories [40].
} 
In particular the large order quotients, $n>4$ turn out to be all quotients of the Schoen manifolds, classified in [40] whose F-theory physics has been investigated in [21].Therefore, the orders of the quotients cannot exceed the symmetries possible in rational elliptic surface and hence these are all possible quotient manifolds ${ }^{26}$ possible via that construction.

Furthermore, the technical results we use to bound MW torsion can also be used to bound the order of fibration-preserving automorphisms of a Calabi-Yau 3-fold. Let $\pi: Y \rightarrow B$ be an elliptic 3-fold given by a Weierstrass equation. Let $L=K(B)$ be the function field of the base and $K=\mathbb{C}(f, g)$ the field generated by the Weierstrass coefficients. Then one of the following must be true:

- $K=\mathbb{C}$. In this case, the fibers in the elliptic curve do not vary, since $f, g$ are constants.

- $L / K$ is a finite field extension. In this case, elementary Galois theory tells us the group of automorphisms of $L$ which fix $f, g$ is contained in $S_{n}$, where $n=[L: K]$. In particular, there are only finitely many automorphisms of the base which fix $f, g$.

- Otherwise, $L$ has transcendence degree 1 over $\mathbb{C}$. In this case, $L$ is the field of meromorphic functions of some Riemann surface $C$, and the inclusion of fields $K \rightarrow L$ means we have a rational map $B \rightarrow C$. Lemma 5.1 below shows that $B$ is flat and $C$ has genus 0 , and so $Y \rightarrow B$ is a special fibration. Resolving indeterminacy of the map $B \rightarrow C$ requires blowing up $B$ in the base points of a pencil of cubics, after which $B$ becomes a rational surface. It is not too hard to show that $Y$ is then Schoen, since the fibration $Y \rightarrow B$ factors through the fiber product $Y \rightarrow \tilde{B} \times_{C} Y_{C}$, and minimality of $Y \rightarrow B$ forces that map to be an isomorphism. Since this construction has been fully analyzed on Schoen manifolds, this shows that any fixed Calabi-Yau 3 -fold admits only finitely many quotient torsors.

\section{Technical results}

Our main result is the bound on torsion groups in smooth Calabi-Yau $n$-folds, $n \geq 3$. The key idea in the proof is the fact that the diagram $(2.4)$ forces $(f, g, \Delta)$ to vanish to order $(4 d, 6 d, 12 d)$ over points in the indeterminacy locus of $\phi$ which obstruct the existence of a flat crepant resolution of the total space. To ease exposition, we will prove the theorem for 3 -folds specifically and comment on its validity for 4 - or 5 -folds at the end.

To avoid having to constantly refer to the diagram, we introduce the phrase "special fibration". An elliptic fibration $Y_{n} \rightarrow B_{n-1}$ is special if it fits into a commutative diagram:

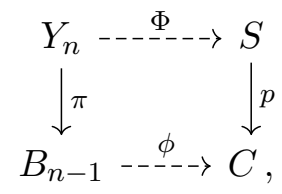

satisfying the following conditions:

\footnotetext{
${ }^{26}$ Alternatives might be quotients of type $\left(K 3 \times T^{k}\right) / \mathbb{Z}_{n}[25,37]$ as well as quotients, that do not require a finite Mordell-Weil group.
} 
- $S \rightarrow C$ is a smooth, minimal elliptic surface, and $C$ is an irreducible curve.

- The horizontal maps are non-constant, rational maps.

- The vertical maps are proper.

- $\phi$ is flat.

\subsection{Global lemmas}

A variety $B$ is rationally connected if two general points $b_{1}, b_{2}$ can be joined by a rational curve, i.e. there exists a regular morphism $\mathbb{P}^{1} \rightarrow B$ taking $[0: 1]$ to $b_{1}$ and $[1: 0]$ to $b_{2}$.

Lemma 5.1. Let $B$ be a rationally connected variety, $C$ an irreducible, separated curve, and $\phi: B \rightarrow C$ a non-constant rational map. Then $C$ has genus 0 and $\phi$ is flat.

Proof. Since $\phi$ is non-constant, we can find points $b_{1}, b_{2} \in B$ such that $\phi\left(b_{1}\right) \neq \phi\left(b_{2}\right)$. Since $B$ is rationally connected, there is a regular map $\mathbb{P}^{1} \rightarrow B$ taking 0 to $b_{1}$ and $\infty$ to $b_{2}$. The composition $\mathbb{P}^{1} \rightarrow C$ is a non-constant rational map, so by [72], ${ }^{27} C$ has genus 0 .

To prove flatness of $\phi$, it suffices to prove that the map is flat over each point in $\mathbb{P}^{1}$. Any proper open neighborhood of $\mathbb{P}^{1}$ has the form $\operatorname{Spec} R_{0}$ for $R_{0}$ a principal ideal domain (PID), so we can determine whether the map is flat by studying the morphism of algebras $R_{0} \rightarrow K(B)$. Since $R_{0}$ is a PID, flatness is equivalent to $K(B)$ being torsion-free, which follows immediately from the fact that the map $B \rightarrow \mathbb{P}^{1}$ is non-constant and $K(B)$ is purely transcendental.

As in section 2, we write $\mathcal{L}_{Y / B}=\left(R^{1} \pi_{*} \mathcal{O}_{Y}\right)^{-1}$ for the fundamental line bundle of the elliptic fibration $\pi: Y \rightarrow B$ and $\omega_{Y}$ (resp. $\omega_{B}$ ) to denote the canonical bundle of $Y$ (resp. $B$ ). A variety $B$ is Fano if $\omega_{B}^{-1}$ is ample. Note that every Fano variety is rationally connected by Theorem 0.1 of [87].

Lemma 5.2. Let $\pi: Y \rightarrow B$ a special elliptic fibration. Then $\mathcal{L}_{Y / B} \cong \phi^{*} \mathcal{L}_{S / C}$.

Proof. The conditions in the definition of a special elliptic fibration allow us to use Prop. III.9.3 in [72] to compute:

$$
\mathcal{L}_{Y / B}=\left(R^{1} \pi_{*} \mathcal{O}_{Y}\right)^{-1}=\left(R^{1} \pi_{*} \Phi^{*} \mathcal{O}_{S}\right)^{-1}=\phi^{*}\left(R^{1} p_{*} \mathcal{O}_{S}\right)^{-1}=\phi^{*} \mathcal{L}_{S / C}
$$

Lemma 5.3. Let $B$ be a rationally connected variety, $\pi: Y \rightarrow B$ be a special fibration and let $d$ be the degree of $\mathcal{L}_{S / \mathbb{P}^{1}}$.

Then $\omega_{Y} \cong \pi^{*}\left(\omega_{B} \otimes \phi^{*}\left(\mathcal{O}_{\mathbb{P}^{1}}(1)\right)^{\otimes d}\right)$.

Proof. This follows from the canonical bundle formula for elliptic fibrations, together with the computation from the previous lemma.

${ }^{27}$ The assertion is proven in IV.2.5.4 and IV.2.5.5. 
Proposition 5.4. Let $\pi: Y \rightarrow B$ be a special elliptic fibration, with $\omega_{Y}$ trivial and $B$ Fano. If $\phi$ is a morphism, then $\operatorname{dim} B=1$.

Proof. First, since $B$ is Fano, $B$ is rationally connected so $C \cong \mathbb{P}^{1}$. Next, since $\pi$ has section, $\pi^{*}: \operatorname{Pic}(B) \rightarrow \operatorname{Pic}(Y)$ is injective, so triviality of $\omega_{Y}$ forces $\phi^{*}\left(\mathcal{O}_{\mathbb{P}^{1}}(1)\right)^{\otimes d} \cong \omega_{B}^{-1}$. Since $B$ is Fano, $\omega_{B}^{-1}$ is ample so $\phi^{*}\left(\mathcal{O}_{\mathbb{P}^{1}}(1)\right)$ is ample.

Finally, suppose $\phi$ is a morphism. Then $\phi^{*}(\mathcal{O}(1))$ is generated by global sections. By Corollary 1.2.15 in [85], $\phi^{*}(\mathcal{O}(1))$ is ample if and only if $\phi$ is finite. Thus, if $\phi$ is a morphism, $\operatorname{dim} B=1$.

In applications, we will be using in the contrapositive of the last proposition, i.e. if $\operatorname{dim} B \geq 2$ then $\phi$ is not a morphism.

\section{$5.2 \quad$ Local lemmas}

In this section $k$ is an algebraically closed field, $R$ a factorial, finitely generated $k$-algebra and $K$ is the fraction field of $R$.

Definition 5.5. Let $\mathfrak{p} \subset R$ be a prime ideal and $I \subset R$ an ideal. The order of vanishing of $I$ at $\mathfrak{p}$ is the largest integer $m$ such that $I \subset \mathfrak{p}^{m}$.

Definition 5.6. Let $\phi=\frac{p}{q} \in K$, with $p, q \in R$ relatively prime. We can think of $\phi$ as a map $\operatorname{Spec} R \rightarrow \mathbb{P}^{1}$. The locus of indeterminacy of $\phi$ is $V(p) \cap V(q) \subset \operatorname{Spec} R$.

Definition 5.7. Let $\phi$ be as above, and assume the locus of indeterminacy of $\phi$ contains a closed point $b$. Let $\mathfrak{m}_{b} \subset R$ be the corresponding ideal. We define $\boldsymbol{m}_{\phi}(\boldsymbol{b})$ to be the order of vanishing of the ideal $(p, q)$ at $\mathfrak{m}_{b}$.

Note that $m_{\phi}(b)$ makes sense whenever we have a rational map $B \rightarrow \mathbb{P}^{1}$ from a normal scheme $B$, since the property is local on $B$. Furthermore, the definition $m_{\phi}(b)$ makes sense for any irreducible component of the indeterminacy locus, and not just for closed points. To ease the exposition, we will refer to irreducible components of the indeterminacy locus as points, although the arguments do not require this.

Proposition 5.8. Let $\phi: B \rightarrow \mathbb{P}^{1}$ be a non-constant rational map and $f$ a global section of $\mathcal{O}_{\mathbb{P}^{1}}(d)$ for some $d>0$. If $b \in B$ is in the indeterminacy locus of $\phi$, then $\phi^{*}(f)$ vanishes to order $m_{\phi}(b) d$ at $b$.

Proof. The claim is local on $B$, so we assume $B$ is affine, say $B=\operatorname{Spec} R$. Choosing coordinates $\left[x_{0}: x_{1}\right]$ on $\mathbb{P}^{1}$, we can express $f\left(x_{0}, x_{1}\right)$ as a homogeneous polynomial of degree $d$ in $x_{0}, x_{1}$. Furthermore, we can write the map $\phi: B \rightarrow \mathbb{P}^{1}$ as $b \mapsto[p(b): q(b)]$, where $p, q \in R$ have no common factors. In this notation, we have $\phi^{*}(f)(b)=f(p(b), q(b))$.

Since $f$ is a homogenous polynomial of degree $d, f \in\left(x_{0}, x_{1}\right)^{d} \subset k\left[x_{0}, x_{1}\right]$. If $b \in B$ is in the locus of indeterminacy of $\phi$, then $\mathfrak{m}_{b}^{m_{\phi}(b)} \supset(p(b), q(b))=\phi^{\#}\left(\left(x_{0}, x_{1}\right)\right)$ so:

$$
\phi^{*} f \in(p(b), q(b))^{d} \subset \mathfrak{m}_{b}^{m_{\phi}(b) d}
$$


We now easily deduce the following:

Corollary 5.9. Let $\pi: Y \rightarrow B$ be a special fibration, let $d$ be the degree of the fundamental line bundle of $\mathcal{L}_{S / \mathbb{P}^{1}}$, and let $b \in B$ be a point in the indeterminacy locus of $\phi$. Then the Weierstrass coefficients $f, g$ of $Y$ vanish to order $(4 n, 6 n)$, where $n=d m_{\phi}(b)$.

Proof. Commutativity of the square (5.1) tells us $f_{B}=\phi^{*}\left(f_{\mathbb{P}}^{1}\right)$ and $g_{B}=\phi^{*}\left(g_{\mathbb{P}}^{1}\right)$. The Weierstrass coefficients of the elliptic surface are homogenous polynomials of degree $4 d, 6 d$ respectively, proving the claim.

Corollary 5.10. Let $\pi: Y \rightarrow B$ be a special elliptic fibration and suppose the order of vanishing of $(f, g)$ does not exceed $(4,6)$ over any point $b \in B$. Then either $\phi$ is a morphism, or $S$ is rational and the locus of poles and the locus of zeros of $\phi$ intersect transversely.

Proof. Assume $\phi$ is not a morphism. Recall that an elliptic surface is rational if and only if the fundamental line bundle has degree 1. If the fundamental line bundle has degree $d>1$, then the order of vanishing over all points in the locus of indeterminacy is at least $(4 d, 6 d)$. The condition on $(f, g)$ forces $d=1$, hence rationality of $S$. If the locus of poles meets the locus of zeros non-transversely at some point $b$, then $m_{\phi}(b)>1$ so $(f, g)$ vanish to order at least $(8,12)$ over $b$.

\subsection{Summary of technical results}

The results of the previous two sections are summarized in the following theorem:

Theorem 5.11. Let $\pi: Y \rightarrow B$ be an elliptic fibration satisfying:

1. $Y$ has trivial canonical bundle.

2. $B$ is a Fano variety of dimension at least 2.

3. The order of vanishing of the Weierstrass coefficients $(f, g)$ does not reach $(8,12)$ over any codimension 2 subvariety of $B$.

If the fibration is special, then:

- $C \cong \mathbb{P}^{1}$

- $S$ is rational, or equivalently $\mathcal{L}_{S / C} \cong \mathcal{O}(1)$.

- $\phi^{*}\left(\mathcal{O}_{\mathbb{P}^{1}}(1)\right) \cong \omega_{B}^{\vee}$

We make some remarks before proving the main theorem.

- When $B$ is a surface, we can use the birational classification of algebraic surfaces to make stronger statements and simplify some of the assumptions. Simply requiring the fibration to not be isotrivial forces the base to be rational. After contracting all exceptional curves in the base, we may assume that $B \cong \mathbb{P}^{2}$ or $\mathbb{F}_{n}$. A computations show that $\phi$ has 8 or 9 points of indeterminacy, with 9 occurring if and only if $B \cong \mathbb{P}^{2}$. 
If we assume that $B=\mathbb{P}^{2}$ e.g., it is easy to see that resolving indeterminacy of $\phi$ means blowing up $\mathbb{P}^{2}$ at the 9 points in the base locus of a pair of cubics, so the new map $\tilde{\phi}: \tilde{B} \rightarrow \mathbb{P}^{1}$ is itself an elliptic fibration. Commutativity of (5.1) gives us a natural map $\tilde{Y} \rightarrow \tilde{B} \times_{\mathbb{P}^{1}} S$. Minimality of the fibration $\tilde{Y} \rightarrow \tilde{B}$ then forces that map to be an isomorphism, showing that any special $Y \rightarrow B$ is birational to a Schoen manifold.

- Requiring the base to be Fano, and more generally requiring $Y \rightarrow B$ to be birational to a fibration over a Fano base, is a mild but necessary requirement for this type of theorem. Even in dimension 2, one has to exclude fibrations of the form $E_{1} \times E_{2} \rightarrow E_{2}$, where $E_{1}, E_{2}$ are elliptic curves, when giving a bound on Mordell-Weil torsion of K3 surfaces. In dimension three, this condition rules out fibrations over Enriques surfaces, which are also isotrivial and thus can have an non-finitely generated MordellWeil group. It also rules fibrations of the form $S \times E \rightarrow \mathbb{P}^{1} \times E$, where $S \rightarrow \mathbb{P}^{1}$ is a K3 surface. However, our conclusion fails in all of these cases, showing the condition is necessary in dimensions 2 and 3.

- By Theorem 1.8 in [66] every $\operatorname{smooth}^{28}$ elliptically fibered Calabi-Yau $n$-fold $Y \rightarrow B$ is birationally equivalent to a (possibly singular) fibration over a Fano base, as long as the original base is not of product type. Roughly speaking, ${ }^{29} B$ is of product type if $B$ is birational to a quotient of a product. This condition is necessary to rule out higher dimensional analogues of isotrivial fibrations in the statement of the boundedness theorem in [66]. However, our theorem also fails for fibrations over a base of product type, and in fact it is easy to construct counterexamples to our theorem in any dimension by taking the product of a K3 with Mordell-Weil group $\mathbb{Z}_{8}$ with a product of elliptic curves.

- The condition on the order of vanishing of $(f, g)$ is precisely the condition needed to guarantee that the Weierstrass model admits a proper, flat crepant resolution, see e.g. [65]. Thus, any smooth fibration $Y \rightarrow B$ is birational to one satisfying the conditions of the previous theorem.

\subsection{Application}

In this section, we prove the following:

Theorem 5.12. Let $\pi: Y \rightarrow B$ be an elliptic fibration satisfying the hypotheses of the previous theorem. Then $M W(Y / B)_{\text {tors }}$ is isomorphic to one of the following groups:

$$
\begin{aligned}
& \mathbb{Z}_{n}: \quad(n=1,2,3,4,5,6), \\
& \mathbb{Z}_{2} \times \mathbb{Z}_{2 m}: \quad(m=1,2), \quad \mathbb{Z}_{3} \times \mathbb{Z}_{3}
\end{aligned}
$$

Note that this list of groups is exactly the list studied in [54], and they can all be realized as torsion subgroups of Schoen manifolds in dimension 3.

\footnotetext{
${ }^{28}$ This condition can be relaxed; see [66] for details.

${ }^{29}$ See [66] for the precise definition.
} 
In order to apply the results of the previous section to rule out the existence of fibrations with a particular torsion group $T$, we need an elliptic surface $S \rightarrow C$ with the following property: for any elliptic fibration $Y \rightarrow B$ whose Mordell-Weil group contains a subgroup isomorphic to $T$, there exists a special diagram:

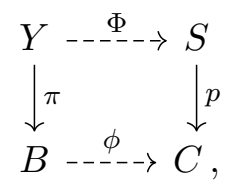

We call $S \rightarrow C$ a universal elliptic surface for $T$.

Proof. If $T$ is cyclic and $|T| \geq 4$, then there is a well-known construction for a universal elliptic surface. We give a construction for $S \rightarrow C$ and $\phi$ in appendix A. For these groups, $C \cong X_{1}(n)$, where $n=|T|$. A computation shows that $C$ has positive genus if $n=11$ or $n \geq 13$, and $\mathcal{L}_{S / C} \cong \mathcal{O}(1)$ for $n=4,5,6$ and $\operatorname{deg} \mathcal{L}_{S / C}>1$ for $n \geq 7$. Thus, we can immediately rule out the existence of a point of order exceeding 6 in $M W(Y / B)$.

If $T$ is not cyclic, then $T$ is isomorphic to $\mathbb{Z}_{m} \times \mathbb{Z}_{n}$ for some pair of positive integers $m, n$ with $1 \neq m \mid n .^{30}$ The previous argument also shows we may assume $n \leq 6$. If $m=n$ and $m \geq 3,{ }^{31}$ then it is also well-known that a surface $S \rightarrow C$ with the desired property exists ([84] Cor. 4.7.2.) and that $C \cong X(m)$. A construction for $S \rightarrow C$ is described in [86]. For these groups, one computes that $X(m)$ has genus 0 if $m=3,4,5$ and has positive genus otherwise. Thus, we can rule out any group containing $\mathbb{Z}_{6} \times \mathbb{Z}_{6}$. A computation using the formulae in appendix B shows that $\mathcal{L}_{S / C} \cong \mathcal{O}(1)$ for $m=3$ and $\operatorname{deg} \mathcal{L}_{S / C}>1$ for $m=4,5$, which allows us to rule out $\mathbb{Z}_{4} \times \mathbb{Z}_{4}$ and $\mathbb{Z}_{5} \times \mathbb{Z}_{5}$.

Finally, we have to rule out $\mathbb{Z}_{2} \times \mathbb{Z}_{6}$ and $\mathbb{Z}_{3} \times \mathbb{Z}_{6} \cdot{ }^{32}$ In appendix $\mathrm{C}$, we explain how to construct surfaces $S \rightarrow C$ for $T \cong \mathbb{Z}_{2} \times \mathbb{Z}_{2 m}(m \geq 2)$ and $T \cong \mathbb{Z}_{m} \times \mathbb{Z}_{2 m}(m \geq 3$ and $m$ odd) with the desired universal property. One can compute the degree of the fundamental line bundle directly from the Weierstrass model obtained from construction, or using the formulae in appendix $\mathrm{B}$, to check that $d=1$ is possible only in the $\mathbb{Z}_{2} \times \mathbb{Z}_{4}$ case.

That will suffice to complete the proof.

Note that when $\operatorname{dim} B \geq 3$, the locus of indeterminacy of $\phi$ still has codimension 2 , since it is a nonempty intersection of 2 hypersurfaces in $B$. Thus, it is impossible to crepantly resolve singularities of the Weierstrass model and obtain a smooth Calabi-Yau total space. We devote appendix A to the explicit construction of Weierstrass models beyond the list above.

\section{Summary and conclusions}

In this work we proved that the Mordell-Weil torsion group $T$ of elliptically fibered CalabiYau $n$-folds, with $n>2$, is no larger than $T=\mathbb{Z}_{6}$ in the cyclic case and $T=\mathbb{Z}_{3} \times \mathbb{Z}_{3}$ or

\footnotetext{
${ }^{30}$ First, note that $\mathbb{Z}_{m} \times \mathbb{Z}_{n} \cong \mathbb{Z}_{\operatorname{gcd}(m, n)} \times \mathbb{Z}_{\mathrm{lcm}(m, n)}$, so we may assume $m \mid n$. Furthermore, we may assume $m \neq 1$, since in that case $T$ is cyclic.

${ }^{31}$ We do not need to consider $\mathbb{Z}_{2} \times \mathbb{Z}_{2}$ since the group is in our list of possible subgroups.

${ }^{32}$ We do not need to rule out $\mathbb{Z}_{2} \times \mathbb{Z}_{4}$, as that group is on our list of subgroups.
} 
$\mathbb{Z}_{2} \times \mathbb{Z}_{4}$ in the general case. We showed explicitly that generic Weierstrass models with higher prescribed torsion have singularities over a codimension two locus in the base which do not admit a crepant resolution. This shows that the list of Weierstrass models that appears in [54] contains every possible torsion group that is allowed on a smooth CalabiYau. Furthermore, we use the same results to bound possible quotient Calabi-Yau torsors as well. We use modular curves to interpret those minimal singularities as a direct feature of the congruence subgroups $\Gamma_{1}(n)$ and $\Gamma(n)$ of $\mathrm{SL}(2, \mathbb{Z})$. There, the amount and type of minimal singularities can directly be read off from cusps in the fundamental domain of the modular curve of the respective congruence subgroup. We find that the torsion groups that can appear on a smooth elliptically fibered Calabi-Yau $d$-fold, with $d \geq 3$, all appear on rational elliptic surfaces. On the other hand, we do not need to worry about crepantly resolving codimension 2 singularities on K3 surfaces, which allows for more possibilities. By connecting the index of the congruence subgroups, with the degree of the fundamental line bundle, we can show however that also K3 cannot exceed $\mathbb{Z}_{8}$ and $\mathbb{Z}_{4} \times \mathbb{Z}_{4}$ as classified in [32]. These K3 manifolds however are all of extremal type and do not admit any free complex structure deformation. Thus, they neither have a stable degeneration limit into rational elliptic surfaces, nor can they be used as building blocks for K3 fibered 3- or 4-folds while preserving the torsion. We further interpret these bounds within the physics of F-theory, where torsion realizes the fundamental group of the global gauge group as a swampland constraint. We argue that, from pure field theory considerations, the above constraints are surprising as it is possible to construct rather large rank $s u(n)$ gauge theories where the massless spectrum respects a putative $\mathbb{Z}_{n}$ quotient factor. This might point towards the possibility, that it is the massive spectrum that does not respect the putative first homotopy group, which is geometrically enforced when torsion is present.

In addition, we observe a variety of surprising effects when large torsion is present that have no field theory explanation yet. The necessity for multiple gauge factors seems especially puzzling from a pure field theory point of view. A possible explanation of this effect might be to view the torsion as a kind orbifold in $\mathrm{SL}(2, \mathbb{Z})$ of the F-theory torus to an $\Gamma_{1}(n)$ subgroup requiring the presence of additional gauge theory sectors for consistency of the modularity. It would also be interesting to explore whether Dai-Freed anomalies [77] could be the right framework to gain a better understanding on why we see these specific gauge algebra factors for a given large fundamental group. These bounds however can partially be avoided in isolated $8 \mathrm{D}$ SUGRA vacua. These vacua admit at most trivial circle compactifications, but none that preserve only a minimal amount of SUSY and the torsion group. They also do not admit a (geometric) heterotic dual due to their rigidity and the fact that their minimal gauge group does not fit into $E_{8} \times E_{8}$ (or $\mathrm{SO}(32)$ ). It would be interesting, to investigate those eight dimensional exceptions from a field theoretical perspective, maybe in the spirit of [76]. Finally, since all admissible torsion groups must be embeddable into a rational elliptic surface (and into its $E_{8}$ lattice) it seems plausible, that the heterotic string plays a similar prominent role as in [69] to explain the constraints we find from the F-theory perspective. 


\section{Acknowledgments}

P. K.O. would like to thank James Gray, Nikhil Raghuram and Fabian Ruehle for interesting discussions. N.H. would like to thank Steve Trettel for helpful explanations. N.H. and P.K.O. would like to thank Dave Morrison and Markus Dierigl for helpful conversations and suggestions. The work of P. K. O. is supported by an individual DFG grant OE 657/1-1. The research of N. H. was partially supported by the National Science Foundation, grant \# PHY-1620842. P. K. O would like to gratefully acknowledge the hospitality of the Simons Center for Geometry and Physics (and the semester long program, The Geometry and Physics of Hitchin Systems) during the completion of this work. The authors would like to express their gratitude towards the anonymous referee for his careful reading and suggestions. The authors would also like to thank the organizers of the 2019 workshop The Physics and Mathematics of F-theory held at Florida State University where this work was initiated.

\section{A Universal elliptic curves with points of order $n \leq 8$}

In this appendix we give explicit constructions for the universal elliptic curves with a point of order $n$, for $2 \leq n \leq 8$, and we show how to extend the natural rational maps $B \rightarrow \mathbb{P}^{1}$ over codimension 1 components of the discriminant when $n=7,8$. The construction for modular curves is well-known in the arithmetic theory of elliptic curves, see [60] See [32, 34] for the generalization to elliptic surfaces. We are including the explicit constructions since these maps have not been used to study higher dimensional elliptic fibrations and the information we need is in the locus of indeterminacy.

The "main" construction applies only to elliptic curves with points of order $n \geq 4$. For completeness, we also explain what happens when we try to parametrize elliptic curves with a 2 or 3 torsion point.

The construction can be carried out over any ground field - for simplicity, we assume our ground $K$ has characteristic 0 throughout.

\section{A.1 $n=2,3$}

Let $E / K$ be an elliptic curve and $P \in E(K)$ a 2-torsion point. We can choose coordinates so:

$$
E: \quad y^{2}=x\left(x^{2}+a x+b\right), \quad P=(0,0),
$$

for some $a, b \in K$. The isomorphism class of the pair $(E, P)$ is determined by $a, b$, but the choice of $a, b$ are not unique. Two choices $a, b$ and $a^{\prime}, b^{\prime}$ give rise to isomorphic pairs if and only if there exists $\lambda \in K^{\times}$such that $\lambda^{2} a=a^{\prime}$ and $\lambda^{4} b=b^{\prime}$. Thus, the modular curve parameterizing pairs $(E, P)$, where $P$ is a 2-torsion point, is:

$$
X_{1}(2)=\left\{(a, b) \in K^{2}\right\} /(a, b) \sim\left(\lambda^{2} a, \lambda^{4} b\right),
$$

Similarly, any pair $(E, P)$ with $P$ a point of order 3 is isomorphic to:

$$
y^{2}+a x y+b y=x^{3}, \quad P=(0,0),
$$


for some $a, b \in K$. Two choices $a, b$ and $a^{\prime}, b^{\prime}$ give rise to isomorphic pairs if and only if there exists $\lambda \in K^{\times}$such that $\lambda a=a^{\prime}$ and $\lambda^{3} b=b^{\prime}$. Thus:

$$
X_{1}(3)=\left\{(a, b) \in K^{2}\right\} /(a, b) \sim\left(\lambda a, \lambda^{3} b\right) .
$$

Both of these quotients are singular - this is due to the presence of finite order points in $\Gamma_{1}(2), \Gamma_{1}(3)$. For $n \geq 4$, there are no more finite order points in the congruence subgroup, so we obtain smooth modular curves. As a result, the moduli spaces are better behaved. This is why we study elliptic curves with a point of order at least 4 separately.

\section{A.2 Universal elliptic curves with a point of order $n \geq 4$}

Let $E / K$ be an elliptic curve and let $P \in E(K)$ be a point which has order at least 4 in the MW group. We can do a change of variable that takes the tangent at $P$ to the line $y=0$. Since the order of $P$ is not 2, the equation of the tangent has the form $y=\lambda x+\nu$, so the equation we obtain after this change of variables only contains "Weierstrass monomials". Explicitly, if the tangent at $P$ is $y=\lambda x+\nu$, then we set $y^{\prime}=y-(\lambda x+\nu)$; setting $y^{\prime}=0$ is then the same as requiring $y=\lambda x+\nu$. Thus, in the new coordinates, the equation of $E$ has the form:

$$
y^{2}+a_{1} x y+a_{3} y=x^{3}+a_{2} x^{2},
$$

since the polynomial on the r.h.s. is the equation of $E$ restricted to the tangent at $(0,0)$, and therefore has to vanish twice at $(0,0)$. Finally, multiplying through by $\frac{a_{2}^{6}}{a_{3}^{6}}$ and replacing $x, y$ by $\frac{a_{3}^{2} x}{a_{2}^{2}}, \frac{a_{3}^{3}}{a_{2}^{3}} y$ gives a Weierstrass equation:

$$
y^{2}+\frac{a_{1} a_{2}}{a_{3}} x y+\frac{a_{2}^{3}}{a_{3}^{2}} y=x^{3}+\frac{a_{2}^{3}}{a_{3}^{2}} x^{2}
$$

The main things to notice about the last equation are that only the coefficients $a_{1}, a_{2}, a_{3}$ are nonzero, and that $a_{2}=a_{3}$.

Thus, for any elliptic curve $E$ with a point $P$ of order at least 4, there exist unique $u, v \in K$ such that the pair $E, P$ is isomorphic to:

$$
y^{2}+(1-u) x y-v y=x^{3}-v x^{2} .
$$

We note that we are using parameters $1-u,-v$ instead of just $u, v$ to simplify future computations.

Viewing this as an elliptic curve over $K(u, v)$, we can compute multiples of $P=(0,0)$ :

$$
\begin{aligned}
& P=(0,0), \quad 2 P=(v, u v), \quad 3 P=(u, v-u), \\
& -P=(0, v), \quad-2 P=(v, 0), \quad-3 P=\left(u, u^{2}\right), \\
& 4 P=\left(\frac{(v-u) v}{u^{2}}, \frac{v^{2}\left(u^{2}+u-v\right)}{u^{3}}\right), \quad 5 P=\left(\frac{u v\left(u^{2}+u-v\right)}{(u-v)^{2}}, \frac{u^{2} v\left(u^{3}+u v-v^{2}\right)}{(u-v)^{3}}\right), \\
& -4 P=\left(\frac{(v-u) v}{u^{2}}, \frac{(v-u)^{2} v}{u^{3}}\right), \quad-5 P=\left(\frac{u v\left(u^{2}+u-v\right)}{(u-v)^{2}}, \frac{v^{2}\left(u^{2}+u-v\right)^{2}}{(u-v)^{3}}\right) .
\end{aligned}
$$


We can construct the universal elliptic curve with an $n$-torsion point for $n=4,5,6$ by setting $P=-3 P, 3 P=-2 P, 3 P=-3 P$, respectively.

$$
\begin{aligned}
& 4 P=O \Longleftrightarrow P=-3 P \Longleftrightarrow u=0, \\
& 5 P=O \Longleftrightarrow 3 P=-2 P \Longleftrightarrow u=v, \\
& 6 P=O \Longleftrightarrow 3 P=-3 P \Longleftrightarrow u^{2}+u=v,
\end{aligned}
$$

This gives us the universal elliptic curves:

$$
\begin{aligned}
y^{2}+x y-t y & =x^{3}-t x^{2}, & & (0,0) \in E[4], \\
y^{2}+(1-t) x y+t y & =x^{3}-t x^{2}, & & (0,0) \in E[5], \\
y^{2}+(1-t) x y-\left(t^{2}+t\right) y & =x^{3}-\left(t^{2}+t\right) x^{2}, & & (0,0) \in E[6]
\end{aligned}
$$

Next, we compute the universal elliptic curves for $n=7,8$. For these fibrations, the relation between $u, v$ will define a singular curve, and we will compute the normalization of the curve explicitly to find the universal curve.

For $n=7$, we set $5 P=-2 P$ to obtain:

$$
7 P=O, \quad \Longleftrightarrow u^{3}-u v+v^{2}=0
$$

The cubic is clearly nodal. The normalization of the curve is:

$$
\operatorname{Spec} \mathbb{C}[t] \rightarrow C^{\prime}=\operatorname{Spec} \mathbb{C}[u, v] / u^{3}-u v+v^{2}, \quad t \mapsto\left(t^{2}-t, t^{3}-t^{2}\right),
$$

and has a rational inverse given by $(u, v) \mapsto \frac{v}{u}$. Thus, the universal elliptic curve is:

$$
y^{2}+\left(1+t-t^{2}\right) x y+\left(t^{2}-t^{3}\right) y=x^{3}+\left(t^{2}-t^{3}\right) x^{2}
$$

For $n=8$, we set $4 P=-4 P$ to obtain:

$$
v\left(u^{2}+u-v\right)=(v-u)^{2} .
$$

The normalization of this curve is:

$$
t \mapsto\left(\frac{(2 t-1)(t-1)}{t},(2 t-1)(t-1)\right)
$$

Note that the inverse of the normalization map is again $(u, v) \mapsto \frac{v}{u}$.

\section{A.2.1 Extending $\phi$}

Let $\pi: Y \rightarrow B$ be an elliptic fibration with an $n$-torsion section $P, 4 \leq n \leq 8$. Let $S \rightarrow \mathbb{P}^{1}$ be the Néron model of one of the following universal elliptic curves over $\mathbb{C}(t)$ :

$$
\begin{aligned}
y^{2}+\left(1+t-t^{2}\right) x y+\left(t^{2}-t^{3}\right) y & =x^{3}+\left(t^{2}-t^{3}\right) x^{2}, \\
y^{2}+\left(t^{2}-(t-1)(2 t-1)\right) x y-t^{3}(t-1)(2 t-1) y & =x^{3}-t^{2}(t-1)(2 t-1) x^{2} .
\end{aligned}
$$


Note that $(0,0)$ has order $n$ where $n=7$ for the first equation and $n=8$ for the second. Let $U=B-V(\Delta)$ and $Y_{U}=Y \times_{B} U$. We have a commutative diagram:

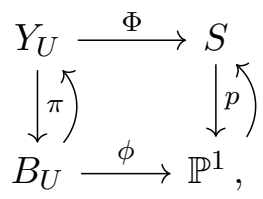

with $B$ flat and non-constant. We can take the closure of the graph to extend to a diagram:

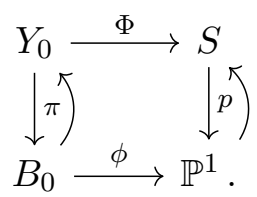

The map $B_{0} \rightarrow \mathbb{P}^{1}$ is obtained by composing the map to the singular modular curve (in $u, v$ coordinates):

$$
b \mapsto\left(\frac{a_{3}-a_{1} a_{2}}{a_{3}}(b), \frac{-a_{2}^{3}}{a_{3}^{2}}(b)\right),
$$

with the inverse of the normalization map. The inverse of the normalization map is the same for both $n=7,8$, and the composite map is:

$$
b \mapsto\left[\frac{a_{3}\left(a_{3}-a_{1} a_{2}\right)}{a_{2}^{3}}(b): 1\right] .
$$

\section{A.3 Weierstrass equation for $\mathbb{Z}_{2} \times \mathbb{Z}_{6}$}

In this section we compute a Weierstrass equation for the elliptic surface with torsion group $\mathbb{Z}_{2} \times \mathbb{Z}_{6}$.

First, fix coordinates $\left[t_{0}: t_{1}\right]$ on $X_{1}(6)$ and compute a Weierstrass model for the universal curve using the methods of the previous section:

$$
y^{2}+\left(t_{1}-t_{0}\right) x y-\left(t_{0} t_{1}+t_{0}^{2}\right) y=x^{3}-t_{1}\left(t_{0} t_{1}+t_{0}^{2}\right) x^{2},
$$

Let $t=\frac{t_{0}}{1}$ be an affine coordinate on $X_{1}(6)$. Note that we have an $I_{1}$ fiber over $t=\frac{-1}{9}$ and an $I_{3}$ fiber over $t=-1$. To construct $X(2,6)$, we take the double cover of $X_{1}(6)$ branched over those two points. Explicitly, the map $X(2,6) \rightarrow X_{1}(6)$ is given by:

$$
\left[u_{0}: u_{1}\right] \mapsto\left[10 u_{1}^{2}-2 u_{0} u_{1}: u_{0}^{2}-9 u_{1}^{2}\right]
$$

Pulling back the Weierstrass equation along this map gives us:

$$
\begin{aligned}
y^{2}+\left(u_{0}^{2}+2 u_{0} u_{1}-19 u_{1}^{2}\right) & x y-\left(10 u_{1}^{2}-2 u_{0} u_{1}\right)\left(u_{0}-u_{1}\right)^{2} y \\
= & x^{3}-\left(10 u_{1}^{2}-2 u_{0} u_{1}\right)\left(u_{0}-u_{1}\right)^{2}\left(u_{0}^{2}-9 u_{1}^{2}\right) x^{2},
\end{aligned}
$$

Now, each coefficient $a_{i}$ is a homogeneous section of degree $2 i$, so the fundamental line bundle of the universal surface over $\mathbb{P}^{1}$ has degree 2 . This degree can also be deduced using (B.16), which we derive in appendix B and by identification of the fundamental line bundle degree as in (3.18). 


\section{A.4 Examples}

In this section, we give Weierstrass models of singular Calabi-Yau 3-folds with Mordell-Weil torsion in $\left\{\mathbb{Z}_{7}, \mathbb{Z}_{8}, \mathbb{Z}_{2} \times \mathbb{Z}_{6}\right\}$. To construct these 3 -folds, we used the method described in the introduction:

- To obtain the Weierstrass model of the modular surface $S \rightarrow \mathbb{P}^{1}$, we use the construction in the universal elliptic curve section.

- We chose the map $\left[s_{0}: s_{1}\right] \times\left[t_{0}: t_{1}\right] \mapsto\left[s_{0} t_{0}: s_{1} t_{1}\right]$ as $\phi$, although any other bi-degree $(1,1)$ map would have given an equivalent 3 -fold. Note that we need a bi-degree $(1,1)$ function because the $\mathcal{L}_{S / \mathbb{P}^{1}}=\mathcal{O}(2)$ for both 7 and 8 torsion. Note that for our choice of $\phi$, the locus of indeterminacy is $[1: 0] \times[0: 1]$ and $[0: 1] \times[1: 0]$.

To obtain the equation for the 3 -fold, we pulled back the sections of the elliptic surface by $\phi$ to obtain the coefficients shown below.

Note that both 3 -folds have $(f, g, \Delta)$ vanishing to order exactly $(8,12,24)$ over $[1$ : $0] \times[0: 1]$ and $[0: 1] \times[1: 0]$, as predicted by our lemma.

\section{A.4.1 Explicit equations}

Explicit equations for the 3 -folds over $\mathbb{P}^{1} \times \mathbb{P}^{1}$ are given below. We use coordinates $\left[s_{0}\right.$ : $\left.s_{1}\right] \times\left[t_{0}: t_{1}\right]$ for the base.

- $\mathbb{Z} / 7$ :

$y^{2}+\left(s_{0}^{2} t_{0}^{2}+s_{0} s_{1} t_{0} t_{1}-s_{1}^{2} t_{1}^{2}\right) x y+s_{0}^{3} s_{1}^{2} t_{0}^{3} t_{1}^{2}\left(s_{0} t_{0}-s_{1} t_{1}\right) y=x^{3}+s_{0} t_{0} s_{1}^{2} t_{1}^{2}\left(s_{0} t_{0}-s_{1} t_{1}\right) x^{2}$,

The coefficients $f, g$ and the discriminant $\Delta=4 f^{3}+27 g^{2}$ are given below:

$$
\begin{aligned}
f= & \frac{1}{48}-\left(s_{0}^{2} t_{0}^{2}-s_{0} s_{1} t_{0} t_{1}+s_{1}^{2} t_{1}^{2}\right) \\
& \times\left(s_{0}^{6} t_{0}^{6}+5 s_{0}^{5} s_{1} t_{0}^{5} t_{1}-10 s_{0}^{4} s_{1}^{2} t_{0}^{4} t_{1}^{2}-15 s_{0}^{3} s_{1}^{3} t_{0}^{3} t_{1}^{3}+30 s_{0}^{2} s_{1}^{4} t_{0}^{2} t_{1}^{4}-11 s_{0} s_{1}^{5} t_{0} t_{1}^{5}+s_{1}^{6} t_{1}^{6}\right) \\
g= & \frac{1}{864} s_{0}^{2} t_{0}^{2}\left(s_{0}^{10} t_{0}^{10}+6 s_{0}^{9} s_{1} t_{0}^{9} t_{1}-15 s_{0}^{8} s_{1}^{2} t_{0}^{8} t_{1}^{2}-46 s_{0}^{7} s_{1}^{3} t_{0}^{7} t_{1}^{3}+174 s_{0}^{6} s_{1}^{4} t_{0}^{6} t_{1}^{4}-222 s_{0}^{5} s_{1}^{5} t_{0}^{5} t_{1}^{5}\right. \\
& \left.+273 s_{0}^{4} s_{1}^{6} t_{0}^{4} t_{1}^{6}-486 s_{0}^{3} s_{1}^{7} t_{0}^{3} t_{1}^{7}+570 s_{0}^{2} s_{1}^{8} t_{0}^{2} t_{1}^{8}-354 s_{0} s_{1}^{9} t_{0} t_{1}^{9}+117 s_{1}^{10} t_{1}^{10}\right) \\
\Delta= & -\frac{1}{16} s_{0}^{7} s_{1}^{7} t_{0}^{7} t_{1}^{7}\left(s_{0} t_{0}-s_{1} t_{1}\right)^{7}\left(s_{0}^{3} t_{0}^{3}+5 s_{0}^{2} s_{1} t_{0}^{2} t_{1}-8 s_{0} s_{1}^{2} t_{0} t_{1}^{2}+s_{1}^{3} t_{1}^{3}\right)
\end{aligned}
$$

- $\mathbb{Z} / 8$ :

$$
\begin{aligned}
& y^{2}+\left(s_{0} s_{1} t_{0} t_{1}+\left(s_{0} t_{0}-s_{1} t_{1}\right)\left(2 s_{1} t_{1}-s_{0} t_{0}\right)\right) x y+s_{0} s_{1}^{3} t_{0} t_{1}^{3}\left(s_{0} t_{0}-2 s_{1} t_{1}\right)\left(s_{1} t_{1}-s_{0} t_{0}\right) y \\
& =x^{3}+s_{1}^{2} t_{1}^{2}\left(s_{0} t_{0}-2 s_{1} t_{1}\right)\left(s_{1} t_{1}-s_{0} t_{0}\right) x^{2}
\end{aligned}
$$


The coefficients $f, g$ and $\Delta$ are:

$$
\begin{aligned}
& f=\frac{1}{48}\left(-s_{0}^{8} t_{0}^{8}+16 s_{0}^{7} s_{1} t_{0}^{7} t_{1}-96 s_{0}^{6} s_{1}^{2} t_{0}^{6} t_{1}^{2}+288 s_{0}^{5} s_{1}^{3} t_{0}^{5} t_{1}^{3}-480 s_{0}^{4} s_{1}^{4} t_{0}^{4} t_{1}^{4}+448 s_{0}^{3} s_{1}^{5} t_{0}^{3} t_{1}^{5}\right. \\
& \left.-224 s_{0}^{2} s_{1}^{6} t_{0}^{2} t_{1}^{6}+64 s_{0} s_{1}^{7} t_{0} t_{1}^{7}-16 s_{1}^{8} t_{1}^{8}\right), \\
& g=\frac{1}{864}\left(s_{0}^{4} t_{0}^{4}-8 s_{0}^{3} s_{1} t_{0}^{3} t_{1}+16 s_{0}^{2} s_{1}^{2} t_{0}^{2} t_{1}^{2}-16 s_{0} s_{1}^{3} t_{0} t_{1}^{3}+8 s_{1}^{4} t_{1}^{4}\right)\left(s_{0}^{8} t_{0}^{8}-16 s_{0}^{7} s_{1} t_{0}^{7} t_{1}\right. \\
& +96 s_{0}^{6} s_{1}^{2} t_{0}^{6} t_{1}^{2}-288 s_{0}^{5} s_{1}^{3} t_{0}^{5} t_{1}^{3}+456 s_{0}^{4} s_{1}^{4} t_{0}^{4} t_{1}^{4}-352 s_{0}^{3} s_{1}^{5} t_{0}^{3} t_{1}^{5}+80 s_{0}^{2} s_{1}^{6} t_{0}^{2} t_{1}^{6} \\
& \left.+32 s_{0} s_{1}^{7} t_{0} t_{1}^{7}-8 s_{1}^{8} t_{1}^{8}\right), \\
& \Delta=\frac{-1}{16} s_{0}^{2} s_{1}^{8} t_{0}^{2} t_{1}^{8}\left(s_{0} t_{0}-2 s_{1} t_{1}\right)^{4}\left(s_{0} t_{0}-s_{1} t_{1}\right)^{8}\left(s_{0}^{2} t_{0}^{2}-8 s_{0} s_{1} t_{0} t_{1}+8 s_{1}^{2} t_{1}^{2}\right) .
\end{aligned}
$$

- $\mathbb{Z}_{2} \times \mathbb{Z}_{6}$ :

$$
\begin{aligned}
& y^{2}+\left(-19 s_{0}^{2} t_{0}^{2}+2 s_{0} s_{1} t_{0} t_{1}+s_{1}^{2} t_{1}^{2}\right) x y+2 s_{0} t_{0}\left(s_{0} t_{0}-s_{1} t_{1}\right)^{2}\left(5 s_{0} t_{0}-s_{1} t_{1}\right)\left(9 s_{0}^{2} t_{0}^{2}-s_{1}^{2} t_{1}^{2}\right) y \\
& =x^{3}+2 s_{0} t_{0}\left(s_{0} t_{0}-s_{1} t_{1}\right)^{2}\left(5 s_{0} t_{0}-s_{1} t_{1}\right) x^{2}, \\
& f=\frac{1}{48}\left(-119761 s_{0}^{8} t_{0}^{8}+36920 s_{0}^{7} s_{1} t_{0}^{7} t_{1}+15700 s_{0}^{6} s_{1}^{2} t_{0}^{6} t_{1}^{2}+11432 s_{0}^{5} s_{1}^{3} t_{0}^{5} t_{1}^{3}-11958 s_{0}^{4} s_{1}^{4} t_{0}^{4} t_{1}^{4}\right. \\
& \left.+1992 s_{0}^{3} s_{1}^{5} t_{0}^{3} t_{1}^{5}+180 s_{0}^{2} s_{1}^{6} t_{0}^{2} t_{1}^{6}-40 s_{0} s_{1}^{7} t_{0} t_{1}^{7}-s_{1}^{8} t_{1}^{8}\right), \\
& g=\frac{1}{864}\left(-41545241 s_{0}^{12} t_{0}^{12}+19809780 s_{0}^{11} s_{1} t_{0}^{11} t_{1}+4915350 s_{0}^{10} s_{1}^{2} t_{0}^{10} t_{1}^{2}+7207028 s_{0}^{9} s_{1}^{3} t_{0}^{9} t_{1}^{3}\right. \\
& -9699039 s_{0}^{8} s_{1}^{4} t_{0}^{8} t_{1}^{4}+2418984 s_{0}^{7} s_{1}^{5} t_{0}^{7} t_{1}^{5}+172820 s_{0}^{6} s_{1}^{6} t_{0}^{6} t_{1}^{6}-12216 s_{0}^{5} s_{1}^{7} t_{0}^{5} t_{1}^{7} \\
& \left.-56679 s_{0}^{4} s_{1}^{8} t_{0}^{4} t_{1}^{8}+12388 s_{0}^{3} s_{1}^{9} t_{0}^{3} t_{1}^{9}-330 s_{0}^{2} s_{1}^{10} t_{0}^{2} t_{1}^{10}-60 s_{0} s_{1}^{11} t_{0} t_{1}^{11}-s_{1}^{12} t_{1}^{12}\right), \\
& \Delta=2 s_{0}^{4} t_{0}^{4}\left(s_{0} t_{0}-s_{1} t_{1}\right)^{6}\left(-5 s_{0} t_{0}+s_{1} t_{1}\right)^{4}\left(-9 s_{0}^{2} t_{0}^{2}+s_{1}^{2} t_{1}^{2}\right)^{2}\left(2969 s_{0}^{6} t_{0}^{6}-318 s_{0}^{5} s_{1} t_{0}^{5} t_{1}\right. \\
& \left.-339 s_{0}^{4} s_{1}^{2} t_{0}^{4} t_{1}^{2}-556 s_{0}^{3} s_{1}^{3} t_{0}^{3} t_{1}^{3}+331 s_{0}^{2} s_{1}^{4} t_{0}^{2} t_{1}^{4}-38 s_{0} s_{1}^{5} t_{0} t_{1}^{5}-s_{1}^{6} t_{1}^{6}\right) \text {. }
\end{aligned}
$$

\section{B Index of congruence subgroups}

In this section, we explain how to compute the indices $[\operatorname{SL}(2, \mathbb{Z}): \Gamma]$ where $\Gamma$ is either one of the congruence subgroups defined earlier or a congruence subgroup associated to a torsion subgroup of the form $\mathbb{Z}_{d} \times \mathbb{Z}_{n d}$. Further details can be found in [73].

\section{B.1 $\Gamma(n)$}

We start by computing the index of $[\operatorname{SL}(2, \mathbb{Z}): \Gamma(n)]$ for $n>1$. The short exact sequence:

$$
0 \longrightarrow \Gamma(n) \longrightarrow \mathrm{SL}(2, \mathbb{Z}) \rightarrow \mathrm{SL}_{2}\left(\mathbb{Z}_{n}\right) \longrightarrow 0,
$$

shows that $[\mathrm{SL}(2, \mathbb{Z}): \Gamma(n)]=\left|\mathrm{SL}_{2}\left(\mathbb{Z}_{n}\right)\right|$. Furthermore, by the Chinese Remainder Theorem, we have isomorphisms:

$$
\mathrm{SL}_{2}\left(\mathbb{Z}_{n m}\right) \rightarrow \mathrm{SL}_{2}\left(\mathbb{Z}_{n}\right) \times \mathrm{SL}_{2}\left(\mathbb{Z}_{m}\right)
$$

whenever $n, m$ are relatively prime. Thus, to compute $[\operatorname{SL}(2, \mathbb{Z}): \Gamma(n)]$ for general $n$, it suffices to find a formula for $\left|\mathrm{SL}_{2}\left(\mathbb{Z}_{n}\right)\right|$ when $n$ is a prime power.

We derive that formula in the following. Assume $n=p^{k}$ for some prime $p$ and some positive integer $k$. We will count the 4 -tuples $(a, b, c, d) \in \mathbb{Z}_{n}^{4}$ with $a d-b c=1$. 
- We count 4-tuples where $a$ is a unit first. There are $p^{k}-p^{k-1}$ units in $\mathbb{Z}_{n}$, so we have that many choices for $a$. Having chosen $a$, we can choose $b, c$ freely, and take $d=\frac{1+b c}{a}$ to guarantee the corresponding matrix has determinant 1. Altogether, there are:

$$
\left(p^{k}-p^{k-1}\right) \cdot p^{k} \cdot p^{k} \cdot 1=p^{3 k}\left(1-\frac{1}{p}\right),
$$

such 4-tuples.

- Next, we count 4 -tuples where $a$ is not a unit. We can choose $a$ in one of $p^{k-1}$ ways. Since $a$ is not a unit, $b, c$ necessarily have to be units. We can choose $b$ freely in the set of units, and we can choose $d$ freely in $\mathbb{Z}_{n}$. We then solve for $c$ as $c=\frac{a d-1}{b}$. This means the number of 4 -tuples is:

$$
p^{k-1} \cdot\left(p^{k}-p^{k-1}\right) \cdot p^{k} \cdot 1=p^{3 k}\left(\frac{1}{p}-\frac{1}{p^{2}}\right),
$$

- Altogether, this means we have:

$$
p^{3 k}\left(\left(1-\frac{1}{p}\right)+\left(\frac{1}{p}-\frac{1}{p^{2}}\right)\right)=p^{3 k}\left(1-\frac{1}{p^{2}}\right),
$$

elements in $\mathrm{SL}_{2}\left(\mathbb{Z}_{n}\right)$.

Finally, if $n=n_{1} \cdots n_{r}$, with $n_{i}=p_{i}^{e_{i}}, p_{i} \neq p_{j}$ for $i \neq j$, then:

$$
[\mathrm{SL}(2, \mathbb{Z}): \Gamma(n)]=\prod_{i}\left[\mathrm{SL}(2, \mathbb{Z}): \Gamma\left(n_{i}\right)\right]=n^{3} \prod_{i} 1-\frac{1}{p_{i}^{2}}
$$

\section{B.2 $\Gamma_{1}(n)$}

Next we use the computation of $[\operatorname{SL}(2, \mathbb{Z}): \Gamma(n)]$ to obtain formulas for the index of $\Gamma_{1}(n)$. Recall that elements of $\Gamma_{1}(n)$, after passing to the quotient, have the form $\left(\begin{array}{ll}1 & a \\ 0 & 1\end{array}\right)$ for some $a \in \mathbb{Z}_{n}$, and that:

$$
\left(\begin{array}{ll}
1 & a \\
0 & 1
\end{array}\right)\left(\begin{array}{ll}
1 & a^{\prime} \\
0 & 1
\end{array}\right)=\left(\begin{array}{cc}
1 & a+a^{\prime} \\
0 & 1
\end{array}\right)
$$

Thus, we have a short exact sequence:

$$
0 \rightarrow \Gamma(n) \rightarrow \Gamma_{1}(n) \rightarrow \mathbb{Z}_{n} \rightarrow 0,
$$

where the map $\Gamma_{1}(n) \rightarrow \mathbb{Z}_{n}$ is the projection onto the top-right factor. This shows that $\left[\Gamma_{1}(n): \Gamma(n)\right]=n$, so we obtain:

$$
\left[\mathrm{SL}(2, \mathbb{Z}): \Gamma_{1}(n)\right]=\frac{1}{n}[\mathrm{SL}(2, \mathbb{Z}): \Gamma(n)]=n^{2} \prod_{p \mid n} 1-\frac{1}{p^{2}} .
$$

We can use this formula to check one of the claims made in the main body - namely, that $24 \mid\left[\operatorname{SL}(2, \mathbb{Z}): \Gamma_{1}(n)\right]$ for $n \geq 5$. By multiplicativity of the index, it suffices to prove the divisibility claim when $n$ is a prime power. 
- First, assume $n=p^{k}$ for $p \geq 5$ and $k \geq 1$. The formula above shows that:

$$
\left[\mathrm{SL}(2, \mathbb{Z}): \Gamma_{1}(n)\right]=p^{3 k}-p^{3 k-2}=p^{3 k-2}(p+1)(p-1) .
$$

Since $3 \bigwedge p, p^{2}-1$ is necessarily divisible by 3 . Furthermore, since $p$ is odd, both $p+1, p-1$ are even and exactly one of them is divisible by 4 . Thus, $p^{2}-1$ is divisible by 3 and 8 , so it is divisible by 24 .

- Next, assume $n=3^{k}$ for $k \geq 2$. Since 3 is odd, $3^{2 k}-1$ is divisible by 8 for the same reason as above. Furthermore, since $k>1,3 k-2 \geq 1$, so the index is divisible by 3 , so again we see that the index is divisible by 24 .

- Finally, assume $n=2^{k}$. Then:

$$
\left[\mathrm{SL}(2, \mathbb{Z}): \Gamma_{1}(n)\right]=2^{3 k}-2^{3 k-2}=2^{3 k-2} \cdot 3
$$

In particular, the index is divisible by 24 precisely when $3 k-2>3$, i.e. $k \geq 3$.

The observations above prove the claim for almost all $n$. We can check the claim by hand for $n=6,12$, since they are not divisible by 8,9 or any larger prime, to complete the argument.

\section{B.3 $\Gamma_{0}(n)$}

While this is not necessary for our proof, we show how to compute $\left[\operatorname{SL}(2, \mathbb{Z}): \Gamma_{0}(n)\right]$ using a similar argument as above. A general element of $\Gamma_{0}(n)$ reduces to a matrix of the form $\left(\begin{array}{ll}a & b \\ 0 & d\end{array}\right)$ in $\mathrm{SL}_{2}\left(\mathbb{Z}_{n}\right)$. The diagonal entries are necessarily units after we reduce $\bmod n$. Now, observe that:

$$
\left(\begin{array}{ll}
a & b \\
0 & d
\end{array}\right)\left(\begin{array}{ll}
a^{\prime} & b^{\prime} \\
0 & d^{\prime}
\end{array}\right)=\left(\begin{array}{cc}
a a^{\prime} & a b^{\prime}+b d^{\prime} \\
0 & d d^{\prime}
\end{array}\right) .
$$

Thus, we can write down a group homomorphism $\Gamma_{0}(n) \rightarrow \mathbb{Z}_{n}^{\times}$whose kernel is exactly $\Gamma_{1}(n)$, so $\left[\Gamma_{0}(n): \Gamma_{1}(n)\right]=\phi(n)$. Using the identity $\phi(n)=n \prod_{p \mid n} 1-\frac{1}{p}$, we obtain:

$$
\left[\mathrm{SL}(2, \mathbb{Z}): \Gamma_{0}(n)\right]=\frac{1}{\phi(n)}\left[\mathrm{SL}(2, \mathbb{Z}): \Gamma_{1}(n)\right]=n \prod_{p \mid n} 1+\frac{1}{p} .
$$

\section{B.4 Non-classical congruence groups}

Finally, we include a formula for the index of the congruence subgroup associated to torsion groups of the form $\mathbb{Z}_{d} \times \mathbb{Z}_{n d}$ with $n, d \geq 2$. With these formulas, we can determine the index of congruence subgroups associated to all finite Abelian groups of length 2, since:

$$
\mathbb{Z}_{m} \times \mathbb{Z}_{n} \cong \mathbb{Z}_{\operatorname{gcd}(m, n)} \times \mathbb{Z}_{\operatorname{lcm}(m, n)},
$$

and clearly $\operatorname{gcd}(m, n) \mid \operatorname{lcm}(m, n)$. 
The idea is to take the fiber product of $X_{1}(n d)$ and $X(d)$. At the level of groups, this corresponds to taking the intersection of the groups. To compute the index of the intersection, we use the identity:

$$
\left[\mathrm{SL}(2, \mathbb{Z}): \Gamma_{1}(n d) \cap \Gamma(d)\right]=\left[\mathrm{SL}(2, \mathbb{Z}): \Gamma_{1}(n d)\right]\left[\Gamma_{1}(n d): \Gamma_{1}(n d) \cap \Gamma(d)\right]
$$

An element of $\Gamma_{1}(n d)$ has the form $\left(\begin{array}{ll}1 & a \\ 0 & 1\end{array}\right) \bmod n d$. In order for it to be an element of $\Gamma(d), a$ has to be a multiple of $d$. Thus, the index of the intersection in $\Gamma_{1}(n d)$ is $n$ so:

$$
\left[\mathrm{SL}(2, \mathbb{Z}): \Gamma_{1}(n d) \cap \Gamma(d)\right]=n^{3} d^{2} \prod_{p \mid n d} 1-\frac{1}{p^{2}}
$$

Thus, the index of the group associated to the Mordell-Weil torsion $\mathbb{Z}_{2} \times \mathbb{Z}_{4}$ has index 24, $\mathbb{Z}_{2} \times \mathbb{Z}_{6}$ has index $48, \mathbb{Z}_{3} \times \mathbb{Z}_{6}$ has index 72 and $\mathbb{Z}_{2} \times \mathbb{Z}_{8}$ has index 96 . Applying the formula for the degree of the fundamental line bundle for $\Gamma_{1}(n)$, this predicts the corresponding universal surface has a fundamental line bundle of degree 1,2,3,4, respectively. These numbers match up exactly with the table in [32].

\section{Universal curves for $\mathbb{Z}_{2} \times \mathbb{Z}_{2 m}$ and $\mathbb{Z}_{m} \times \mathbb{Z}_{2 m}$}

\section{C.1 Set-up}

Let $T$ be a group in one of the following families:

$$
\begin{array}{ll}
\mathbb{Z}_{2} \times \mathbb{Z}_{2 m} & (m \geq 2), \\
\mathbb{Z}_{m} \times \mathbb{Z}_{2 m} & (m \geq 3) .
\end{array}
$$

We construct an elliptic surface $S^{\prime} \rightarrow C^{\prime}$ which has the following properties:

- $S^{\prime} \rightarrow C^{\prime}$ comes with morphisms making the following square commute:

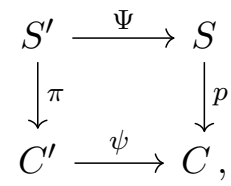

where $S \rightarrow C$ is the universal surface for $\mathbb{Z}_{2 m}$ if $T \cong \mathbb{Z}_{2} \times \mathbb{Z}_{2 m}$, and $S \rightarrow C$ is the universal surface for $\mathbb{Z}_{m} \times \mathbb{Z}_{m}$ if $T \cong \mathbb{Z}_{m} \times \mathbb{Z}_{2 m}$. Furthermore, $\psi$ has degree 2 if $T \cong \mathbb{Z}_{2} \times \mathbb{Z}_{2 m}$, and degree 3 if $T \cong \mathbb{Z}_{m} \times \mathbb{Z}_{2 m}$.

- If $Y \rightarrow B$ is any elliptic fibration with $M W(Y / B)_{\text {tors }}$ containing a subgroup isomorphic to $T$, then the diagram:

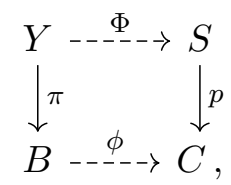


factors through:

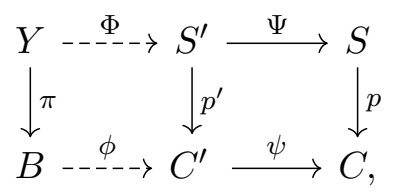

\section{C.2 Construction}

Let $k$ be the function field of $C$, and fix a short Weierstrass equation for the generic fiber of $S \rightarrow C$ :

$$
E \quad y^{2}=x^{3}+f x+g \quad(f, g \in k) .
$$

We construct a field extension $k^{\prime} / k$ :

$$
k^{\prime}=\left\{\begin{array}{c}
k\left(\sqrt{-16\left(4 f^{3}+27 g^{2}\right)}\right) \text { if } T \cong \mathbb{Z}_{2} \times \mathbb{Z}_{2 m} \\
k[x] /\left(x^{3}+f x+g\right) \text { if } T \cong \mathbb{Z}_{m} \times \mathbb{Z}_{2 m}
\end{array}\right.
$$

Let $C^{\prime}$ be the algebraic curve with function field $k^{\prime}$, let $\psi: C^{\prime} \rightarrow C$ be corresponding morphism of curves and let $S^{\prime}=S \times_{C} C^{\prime}$ be the fiber product.

Clearly $\psi$ has degree 2 if $C \cong X_{1}(2 m)$ and degree 3 if $C \cong X(m)$. All that we need to show is that the universal map $B \rightarrow C$ factors appropriately. For that, we use the following well-known facts from Galois theory:

Lemma C.1. Let $k$ be a field of characteristic not equal to 2 or $3, q(x)=x^{3}+f x+g \in k[x]$ and $\Delta=-16\left(4 f^{3}+27 g^{2}\right)$ the discriminant of $q$. We assume that $q(x)$ has distinct roots in the algebraic closure of $k$, or equivalently, that $\Delta \neq 0$. Let $K / k$ be an arbitrary field extension. Then:

1. Assume $q$ has a root in $k$ and splits completely in $K$. Then $K$ contains a subfield isomorphic to $k(\sqrt{\Delta})$.

2. Assume $q$ is irreducible over $k$ and has a root in $K$. Then $K$ contains a subfield isomorphic to $k[x] /(q(x))$.

If $C \cong X_{1}(2 m)$, then the generic fiber has exactly one point of order 2 defined over $k$, so (C.6) has exactly one root in $k$. For any elliptic fibration $Y \rightarrow B$ with Mordell-Weil torsion subgroup $T$, we have a map $\phi: B \rightarrow X_{1}(2 m)$. This induces an inclusion of function fields $k \rightarrow K(B)$. We will identify $k$ with its image in $K(B)$.

Now, since $Y / B$ has all of its 2-torsion points defined over $K(B)$, the polynomial:

$$
x^{3}+f x+\phi^{*}(g),
$$

in (C.6) splits completely over $K(B)$. Thus, $K(B)$ contains a subfield isomorphic to $k(\sqrt{\Delta})$, so we have an inclusion of fields $k \subset k(\sqrt{\Delta}) \subset K(B)$. This shows that the $B \rightarrow C$ factors through $C^{\prime} \rightarrow C$ as claimed.

Similarly, if $C \cong X(m)$, with $m$ odd, then the cubic:

$$
x^{3}+f x+g
$$


is irreducible over $k$. If we have a fibration $Y \rightarrow B$ with $M W(Y / B)$ containing a subgroup isomorphic to $\mathbb{Z}_{m} \times \mathbb{Z}_{2 m}$, then the cubic acquires a root in $K(B)$, so $K(B)$ contains a subfield isomorphic to $k[x] /\left(x^{3}+f x+g\right)$.

Thus, $C^{\prime}$ has the properties we claim it does. Applying these results to the groups $\mathbb{Z}_{2} \times \mathbb{Z}_{6}$ and $\mathbb{Z}_{3} \times \mathbb{Z}_{6}$, we obtain an algebraic proof that the associated universal surfaces have fundamental line bundles of degree 2, 3, respectively. This is consistent with the degrees we computed using the index formula, and with the computations by Miranda [46].

\section{C.3 $\mathbb{Z}_{2} \times \mathbb{Z}_{4}$}

If we apply the construction to $X_{1}(4)$ using the model computed above, we obtain an equation for an elliptic surface $S \rightarrow C$ with $\mathcal{L}_{S / C}=\mathcal{O}_{C}(2)$. However, since the universal surface over $X_{1}(4)$ has an $I_{1}^{*}$ fiber, $S \rightarrow C$ has a nonminimal ${ }^{33}$ singularity over the $I_{1}^{*}$ fiber. When we run Tate's algorithm to compute the Néron model of $S \rightarrow C$, that singularity is replaced by an $I_{2}$ fiber and the degree of the fundamental line bundle drops by 1 . One can also check this using the formulae in appendix B.

Open Access. This article is distributed under the terms of the Creative Commons Attribution License (CC-BY 4.0), which permits any use, distribution and reproduction in any medium, provided the original author(s) and source are credited.

\section{References}

[1] C. Vafa, The string landscape and the swampland, hep-th/0509212 [INSPIRE].

[2] E. Palti, The swampland: introduction and review, Fortsch. Phys. 67 (2019) 1900037 [arXiv: 1903.06239] [INSPIRE].

[3] N. Arkani-Hamed, L. Motl, A. Nicolis and C. Vafa, The string landscape, black holes and gravity as the weakest force, JHEP 06 (2007) 060 [hep-th/0601001] [INSPIRE].

[4] S.-J. Lee, W. Lerche and T. Weigand, Tensionless strings and the weak gravity conjecture, JHEP 10 (2018) 164 [arXiv:1808.05958] [INSPIRE].

[5] H. Ooguri and C. Vafa, On the geometry of the string landscape and the swampland, Nucl. Phys. B 766 (2007) 21 [hep-th/0605264] [INSPIRE].

[6] D. Klaewer and E. Palti, Super-Planckian spatial field variations and quantum gravity, JHEP 01 (2017) 088 [arXiv:1610.00010] [INSPIRE].

[7] T.W. Grimm, E. Palti and I. Valenzuela, Infinite distances in field space and massless towers of states, JHEP 08 (2018) 143 [arXiv: 1802.08264] [INSPIRE].

[8] C. Mayrhofer, E. Palti and T. Weigand, U(1) symmetries in F-theory GUTs with multiple sections, JHEP 03 (2013) 098 [arXiv:1211.6742] [INSPIRE].

[9] V. Braun, T.W. Grimm and J. Keitel, Geometric engineering in toric F-theory and GUTs with U(1) gauge factors, JHEP 12 (2013) 069 [arXiv: 1306.0577] [INSPIRE].

[10] M. Cvetič, D. Klevers and H. Piragua, F-theory compactifications with multiple U(1)-factors: constructing elliptic fibrations with rational sections, JHEP 06 (2013) 067 [arXiv: 1303.6970] [INSPIRE].

\footnotetext{
${ }^{33}$ Precisely, we mean there is a point in $C$ where $(f, g)$ vanish to order $(4,6)$.
} 
[11] D.S. Park and W. Taylor, Constraints on 6D supergravity theories with Abelian gauge symmetry, JHEP 01 (2012) 141 [arXiv:1110.5916] [INSPIRE].

[12] C. Lawrie, S. Schäfer-Nameki and J.-M. Wong, F-theory and all things rational: surveying U(1) symmetries with rational sections, JHEP 09 (2015) 144 [arXiv: 1504.05593] [INSPIRE].

[13] N. Raghuram, Abelian F-theory models with charge-3 and charge-4 matter, JHEP 05 (2018) 050 [arXiv: 1711.03210$]$ [INSPIRE].

[14] N. Raghuram and W. Taylor, Large U(1) charges in F-theory, JHEP 10 (2018) 182 [arXiv: 1809.01666] [INSPIRE].

[15] W. Taylor and A.P. Turner, An infinite swampland of $\mathrm{U}(1)$ charge spectra in $6 D$ supergravity theories, JHEP 06 (2018) 010 [arXiv:1803.04447] [INSPIRE].

[16] F.M. Cianci, D.K. Mayorga Peña and R. Valandro, High U(1) charges in type IIB models and their F-theory lift, JHEP 04 (2019) 012 [arXiv: 1811.11777] [INSPIRE].

[17] A. Collinucci, M. Fazzi, D.R. Morrison and R. Valandro, High electric charges in M-theory from quiver varieties, JHEP 11 (2019) 111 [arXiv:1906.02202] [INSPIRE].

[18] M. Del Zotto, J.J. Heckman, D.R. Morrison and D.S. Park, 6D SCFTs and gravity, JHEP 06 (2015) 158 [arXiv: 1412.6526] [inSPIRE].

[19] L.B. Anderson, A. Grassi, J. Gray and P.-K. Oehlmann, F-theory on quotient threefolds with $(2,0)$ discrete superconformal matter, JHEP 06 (2018) 098 [arXiv: 1801.08658] [INSPIRE].

[20] W. Taylor and Y.-N. Wang, The F-theory geometry with most flux vacua, JHEP 12 (2015) 164 [arXiv: 1511.03209] [INSPIRE].

[21] L.B. Anderson, J. Gray and P.-K. Oehlmann, F-theory on quotients of elliptic Calabi-Yau threefolds, JHEP 12 (2019) 131 [arXiv:1906.11955] [INSPIRE].

[22] R. Donagi, B.A. Ovrut, T. Pantev and D. Waldram, Standard models from heterotic M-theory, Adv. Theor. Math. Phys. 5 (2002) 93 [hep-th/9912208] [INSPIRE].

[23] M. Bershadsky, T. Pantev and V. Sadov, F-theory with quantized fluxes, Adv. Theor. Math. Phys. 3 (1999) 727 [hep-th/9805056] [INSPIRE].

[24] P. Berglund, A. Klemm, P. Mayr and S. Theisen, On type IIB vacua with varying coupling constant, Nucl. Phys. B 558 (1999) 178 [hep-th/9805189] [INSPIRE].

[25] C.F. Cota, A. Klemm and T. Schimannek, Topological strings on genus one fibered Calabi-Yau 3-folds and string dualities, JHEP 11 (2019) 170 [arXiv:1910.01988] [INSPIRE].

[26] V. Braun and D.R. Morrison, F-theory on genus-one fibrations, JHEP 08 (2014) 132 [arXiv: 1401.7844] [INSPIRE].

[27] L.B. Anderson, I. García-Etxebarria, T.W. Grimm and J. Keitel, Physics of F-theory compactifications without section, JHEP 12 (2014) 156 [arXiv:1406.5180] [INSPIRE].

[28] V. Braun, T.W. Grimm and J. Keitel, Complete intersection fibers in F-theory, JHEP 03 (2015) 125 [arXiv:1411.2615] [INSPIRE].

[29] C. Mayrhofer, E. Palti, O. Till and T. Weigand, Discrete gauge symmetries by Higgsing in four-dimensional F-theory compactifications, JHEP 12 (2014) 068 [arXiv:1408.6831] [INSPIRE].

[30] C. Mayrhofer, E. Palti, O. Till and T. Weigand, On discrete symmetries and torsion homology in F-theory, JHEP 06 (2015) 029 [arXiv: 1410.7814] [INSPIRE]. 
[31] D.R. Morrison and W. Taylor, Sections, multisections and U(1) fields in F-theory, arXiv: 1404.1527 [INSPIRE].

[32] R. Miranda and U. Persson, Torsion groups of elliptic surfaces, Compos. Math. 72 (1989) 3.

[33] T. Shioda, On elliptic modular surfaces, J. Math. Soc. Japan 24 (1972) 20.

[34] M. Schütt and T. Shioda, Elliptic surfaces, in Algebraic geometry in East Asia - Seoul 2008, Adv. Stud. Pure Math. 60 (2010) 51 [arXiv:0907.0298].

[35] T. Shioda, Mordell-Weil lattices for higher genus fibration over a curve, in New trends in algebraic geometry, London Math. Soc. Lect. Note Ser. 264 (1999) 359.

[36] R. Wazir, Arithmetic on elliptic threefolds, Compos. Math. 140 (2004) 567 [math.NT/0112259].

[37] J. de Boer et al., Triples, fluxes and strings, Adv. Theor. Math. Phys. 4 (2002) 995 [hep-th/0103170] [INSPIRE].

[38] L. Bhardwaj, M. Del Zotto, J.J. Heckman, D.R. Morrison, T. Rudelius and C. Vafa, F-theory and the classification of little strings, Phys. Rev. D 93 (2016) 086002 [Erratum ibid. D 100 (2019) 029901] [arXiv:1511.05565] [INSPIRE].

[39] L.B. Anderson, J. Gray and B. Hammack, Fibrations in non-simply connected Calabi-Yau quotients, JHEP 08 (2018) 128 [arXiv: 1805.05497] [INSPIRE].

[40] V. Bouchard and R. Donagi, On a class of non-simply connected Calabi-Yau threefolds, Commun. Num. Theor. Phys. 2 (2008) 1 [arXiv:0704.3096] [INSPIRE].

[41] M. Gross, A finiteness theorem for elliptic Calabi-Yau threefolds, alg-geom/9305002 [INSPIRE].

[42] C. Schoen, On fiber products of rational elliptic surfaces with section, Math. Z. 197 (1988) 177 .

[43] P. Candelas, A.M. Dale, C.A. Lütken and R. Schimmrigk, Complete intersection Calabi-Yau manifolds, Nucl. Phys. B 298 (1988) 493 [INSPIRE].

[44] R. Donagi, B.A. Ovrut, T. Pantev and D. Waldram, Standard Model bundles on nonsimply connected Calabi-Yau threefolds, JHEP 08 (2001) 053 [hep-th/0008008] [INSPIRE].

[45] A.P. Braun, C.R. Brodie and A. Lukas, Heterotic line bundle models on elliptically fibered Calabi-Yau three-folds, JHEP 04 (2018) 087 [arXiv: 1706.07688] [INSPIRE].

[46] R. Miranda, Elliptic surfaces and the Mordell-Weil group, in XII school of algebra, part I (Diamantina, Minas Gerais, Brazil, 1992), Mat. Contemp. 6 (1994) 147.

[47] D.R. Morrison and D.S. Park, F-theory and the Mordell-Weil group of elliptically-fibered Calabi-Yau threefolds, JHEP 10 (2012) 128 [arXiv:1208.2695] [INSPIRE].

[48] D.S. Park, Anomaly equations and intersection theory, JHEP 01 (2012) 093 [arXiv:1111.2351] [INSPIRE].

[49] M. Cvetič and L. Lin, The global gauge group structure of F-theory compactification with $\mathrm{U}(1) s$, JHEP 01 (2018) 157 [arXiv: 1706.08521] [INSPIRE].

[50] T.W. Grimm, A. Kapfer and D. Klevers, The arithmetic of elliptic fibrations in gauge theories on a circle, JHEP 06 (2016) 112 [arXiv:1510.04281] [INSPIRE].

[51] M. Cvetič and L. Lin, TASI lectures on Abelian and discrete symmetries in F-theory, PoS (TASI2017)020 (2018) [arXiv:1809.00012] [INSPIRE]. 
[52] C. Mayrhofer, D.R. Morrison, O. Till and T. Weigand, Mordell-Weil torsion and the global structure of gauge groups in F-theory, JHEP 10 (2014) 016 [arXiv:1405.3656] [INSPIRE].

[53] Y. Kimura, Gauge symmetries and matter fields in F-theory models without section compactifications on double cover and Fermat quartic K3 constructions times K3, Adv. Theor. Math. Phys. 21 (2017) 2087 [arXiv:1603.03212] [InSPIRE].

[54] P.S. Aspinwall and D.R. Morrison, Nonsimply connected gauge groups and rational points on elliptic curves, JHEP 07 (1998) 012 [hep-th/9805206] [INSPIRE].

[55] M. Esole, M.J. Kang and S.-T. Yau, Mordell-Weil torsion, anomalies and phase transitions, arXiv: 1712.02337 [INSPIRE].

[56] P. Griffiths and J. Harris, Principles of algebraic geometry, John Wiley \& Sons Inc., U.S.A. (1994).

[57] D. Klevers, D.K. Mayorga Pena, P.-K. Oehlmann, H. Piragua and J. Reuter, F-theory on all toric hypersurface fibrations and its Higgs branches, JHEP 01 (2015) 142 [arXiv:1408.4808] [INSPIRE].

[58] P.-K. Oehlmann, J. Reuter and T. Schimannek, Mordell-Weil torsion in the mirror of multi-sections, JHEP 12 (2016) 031 [arXiv:1604.00011] [INSPIRE].

[59] A. Braun, A. Lukas and C. Sun, Discrete symmetries of Calabi-Yau hypersurfaces in toric four-folds, Commun. Math. Phys. 360 (2018) 935 [arXiv: 1704.07812] [INSPIRE].

[60] B. Mazur and D. Goldfeld, Rational isogenies of prime degree, Invent. Math. 44 (1978) 129.

[61] I. Shimada, On elliptic K3 surfaces, Michigan Math. J. 47 (2000) 423 [math. AG/0505140].

[62] K. Oguiso, On algebraic fiber space structures on a Calabi-Yau 3-fold, Int. J. Math. 04 (1993) 439.

[63] U. Persson, Configurations of Kodaira fibers on rational elliptic surfaces, Math. Z. 205 (1990) 1.

[64] K. Oguiso and T. Shioda, The Mordell-Weil lattice of a rational elliptic surface, Comment. Math. Univ. St. Paul. 40 (1991) 83.

[65] A. Grassi, Log contractions and equidimensional models of elliptic threefolds, J. Alg. Geom. 4 (1995) 255 [alg-geom/9305003].

[66] G. Di Cerbo and R. Svaldi, Birational boundedness of low dimensional elliptic Calabi-Yau varieties with a section, arXiv:1608.02997.

[67] D.R. Morrison, D.S. Park and W. Taylor, Non-Higgsable Abelian gauge symmetry and F-theory on fiber products of rational elliptic surfaces, Adv. Theor. Math. Phys. 22 (2018) 177 [arXiv: 1610.06929] [INSPIRE].

[68] C. Vafa, Evidence for F-theory, Nucl. Phys. B 469 (1996) 403 [hep-th/9602022] [INSPIRE].

[69] S.-J. Lee and T. Weigand, Swampland bounds on the Abelian gauge sector, Phys. Rev. D 100 (2019) 026015 [arXiv: 1905.13213] [INSPIRE].

[70] S.H. Katz and C. Vafa, Matter from geometry, Nucl. Phys. B 497 (1997) 146 [hep-th/9606086] [INSPIRE].

[71] O. Aharony, N. Seiberg and Y. Tachikawa, Reading between the lines of four-dimensional gauge theories, JHEP 08 (2013) 115 [arXiv:1305.0318] [INSPIRE]. 
[72] R. Hartshorne, Algebraic geometry, Grad. Texts Math. 52, Springer-Verlag, New York, NY, U.S.A. (1977).

[73] F. Diamond and J. Shurman, A first course in modular forms, Grad. Texts Math. 228, Springer, New York, NY, U.S.A. (2005).

[74] D.A. Cox and W.R. Parry, Torsion in elliptic curves over k(t), Compos. Math. 41 (1980) 3.

[75] A. Font, I. Garcia-Etxebarria, D. Lüst, S. Massai and C. Mayrhofer, Non-geometric heterotic backgrounds and 6 D SCFTs/LSTs, PoS(CORFU2016) 123 (2017) [arXiv:1712.07083] [INSPIRE].

[76] I. García-Etxebarria, H. Hayashi, K. Ohmori, Y. Tachikawa and K. Yonekura, $8 d$ gauge anomalies and the topological Green-Schwarz mechanism, JHEP 11 (2017) 177 [arXiv: 1710.04218] [INSPIRE].

[77] I. García-Etxebarria and M. Montero, Dai-Freed anomalies in particle physics, JHEP 08 (2019) 003 [arXiv: 1808.00009] [INSPIRE].

[78] R. Miranda, Persson's list of singular fibers for a rational elliptic surface, Math. Z. 205 (1990) 191.

[79] U. Persson, Configurations of Kodaira fibers on rational elliptic surfaces, Math. Z. 205 (1990) 1.

[80] I. Shimada, On elliptic K3 surfaces, Michigan Math. J. 47 (2000) 423.

[81] D. Huybrechts, Lectures on K3 surfaces, Cambridge University Press, Cambridge, U.K. (2016).

[82] W.A. Stein et al., Sage mathematics software (version 4.7), the Sage Development Team, http://www.sagemath.org, (2019).

[83] C. Kurth and L. Long, Computations with finite index subgroups of $\mathrm{PSL}_{2}(Z)$ using Farey symbols, arXiv:0710.1835.

[84] N.M. Katz and B. Mazur, Arithmetic moduli of elliptic curves, Annals Math. Stud. 108, Princeton University Press, Princeton, NJ, U.S.A. (1985).

[85] R. Lazarsfeld, Positivity in algebraic geometry. I, Ergeb. Math. Grenz. 48, Springer-Verlag, Berlin, Germany (2004).

[86] T. Shioda, On elliptic modular surfaces, J. Math. Soc. Japan 24 (1972) 20.

[87] J. Kollár, Y. Miyaoka and S. Mori, Rational connectedness and boundedness of Fano manifolds, J. Diff. Geom. 36 (1992) 765.

[88] P. Arras, A. Grassi and T. Weigand, Terminal singularities, Milnor numbers and matter in F-theory, J. Geom. Phys. 123 (2018) 71 [arXiv:1612.05646] [INSPIRE].

[89] A. Grassi and T. Weigand, On topological invariants of algebraic threefolds with (Q-factorial) singularities, arXiv:1804.02424 [INSPIRE]. 